\title{
On the steady streaming flow due to high-frequency vibration in nearly inviscid liquid bridges
}

\author{
By JOSÉ A. NICOLÁS, DAMIÁN RIVAS \\ AND JOSÉ M. VEGA \\ Departamento de Fundamentos Matemáticos, Universidad Politécnica de Madrid, \\ Plaza Cardenal Cisneros, 3, 28040 Madrid, Spain
}

The steady streaming flow due to vibration in capillary bridges is considered in the limiting case when both the capillary Reynolds number and the non-dimensional vibration frequency (based on the capillary time) are large. An asymptotic model is obtained that provides the streaming flow in the bulk, outside the thin oscillatory boundary layers near the disks and the interface. Numerical integration of this model shows that several symmetric and non-symmetric streaming flow patterns are obtained for varying values of the vibration parameters. As a by-product, the quantitative response of the liquid bridge to high-frequency axial vibrations of the disks is also obtained.

\section{Introduction}

Thermocapillary flows are of inherent interest in a variety of fields, for example in pattern formation (Dubois et al. 1992; Vince \& Dubois 1992; Daviaud \& Vince 1993). Also, in microgravity conditions buoyancy is essentially absent and thermocapillary stress provides the main mechanism to maintain convection in non-isothermal conditions. The resulting (steady or non-steady) thermocapillary flow is always present in the melt when applying the float-zone technique for unidirectional semiconductor crystal growth, and promotes undesirable non-uniformities in dopant distribution and crystal striations (Jurish \& Löser 1990). In order to improve crystal quality, several methods have been suggested to minimize thermocapillary convection, namely floatzone rotation (Fowlis \& Roberts 1986), application of magnetic fields (Robertson \& O'Connor 1988; Cröll, Dold \& Benz 1994) or gas jets (Dressler \& Sivakumaran 1988), coating or partial covering of the melt (Lan \& Kou 1991) and, more recently, controlled vibration of the float zone (Anilkumar et al. 1993).

The last above-mentioned method is based on the well-known fact that mechanical vibrations produce steady (or slowly varying) flows through parametric resonance effects. As pointed out by Anilkumar et al. (1993), a good understanding of the streaming flow due to vibration is essential before considering its combined effect with thermocapillary stress. The main object of this paper is to analyse precisely the qualitative and quantitative properties of the streaming flow in nearly inviscid capillary bridges in one of the three distinguished limits (in fact, the more interesting one) that must be analysed separately, as will be explained below.

Streaming flows produced by mechanical vibrations in finite geometries such as 
liquid bridges or liquid-filled containers are receiving an increasing interest in the literature. But most works either dealt with fluid configurations without interfaces (Zapyanov, Kozhoukharova \& Iordanova 1988; Biringen \& Peltier 1990; Riley 1992; Yan, Ingham \& Morton 1993; Faroog \& Homsy 1994), or were modelled phenomenologically (Lee. Anilkumar \& Wang 1996), or were based on a strictly inviscid analysis (Pierce \& Knobloch 1994, and references given therein); the latter leads to the well-known (Davey \& Stewartson 1974) models which ultimately depend on initial conditions (Knobloch \& Pierce 1997). This has been so in spite of the fact that Longuet-Higgins (1953), Phillips (1977) and Craik (1982) subsequently pointed out several interesting basic ideas on the role of both the free-surface deformation and viscosity (however small viscosity may be) on the generation of streaming flows. But their ideas do not seem to have been followed systematically to obtain asymptotically correct models that account for the structure of these flows in finite geometries when free surfaces are present. Our previous work (Nicolás \& Vega 1996; Nicolás, Rivas \& Vega 1997) and this paper amount to a systematic attempt in this direction, and cover two of the three distinguished asymptotic limits that must be analysed separately because both the physical mechanisms driving the streaming flow and the structure of the leading oscillating flow are essentially different in each case. These limits are explained below in terms of the liquid bridge geometry but they are not dependent on this particular geometry and apply to other configurations as well. Here we focus on the liquid bridge configuration because we are primarily interested in the practical application mentioned above, but the extension of the analysis below to related problems (e.g. water waves in vibrated finite containers) is quite straightforward. The main underlying ideas are expected to apply also to not so closely related problems. For instance, recent experimental work by Weidman et al. (1997) has shown the appearance of novel two-dimensional vortical structures in vertically vibrated horizontal soap films. These patterns seem to be associated with secondary streaming motions like those analysed below.

If density is assumed to be strictly constant, the driving mechanism yielding streaming flows essentially comes from vorticity-induced phase lags between the velocity components that lead to a rectification of the oscillation. In capillary bridges the mechanism depends on the orders of magnitude of the capillary or modified Reynolds number, $C^{-1}=(\rho \sigma R)^{1 / 2} / \mu$ (with $\rho=$ density, $\sigma=$ surface tension, $\mu=$ viscosity and $R=$ radius of the disks supporting the bridge) and the non-dimensional vibrating frequency, $\Omega=\tilde{\Omega}\left(\rho R^{3} / \sigma\right)^{1 / 2}$ (where $\tilde{\Omega}$ is the dimensional frequency). If in addition, $C^{-1} \gg 1$ (nearly inviscid limit) then vorticity is mainly confined to thin viscous boundary layers in the oscillatory velocity field. The Stokes boundary layers near the disks produce a steady (or slowly varying) tangential forcing velocity at the edge of the layers that was first calculated by Schlichting (1932), and the boundary layer near the interface produces a tangential stress that seems to have been first considered by Longuet-Higgins (1953). Since these two forcing mechanisms are tangential to the edge of the oscillatory boundary layers, viscous effects (providing vorticity diffusion) cannot be ignored when calculating the streaming flow in the bulk, however large the effective Reynolds number associated with this flow may be. The streaming flow provides the main mechanism for vorticity transport from the oscillatory boundary layers to the bulk. Notice that the forcing tangential stress at the edge of the free surface is a priori the best mechanism to counterbalance thermocapillary stress (which is also a forcing tangential stress at the free surface); the forcing tangential velocity near the disks on the other hand is an undesirable by-product. Three distinguished limits must be considered separately, 
depending on the order of magnitude of the non-dimensional vibrating frequency $\Omega$ :

(A) If $\Omega \sim 1$ or, more generally, if $C \ll \Omega \ll C^{-1}$, then the steady streaming flow exhibits a large variety of qualitatively different patterns, depending on (i) the orders of magnitude of the forcing frequencies and amplitudes, (ii) whether $\Omega$ is close to a natural inviscid frequency or not, and (iii) whether only one disk is vibrated or both disks are; when both disks are vibrated (always with the same frequency) the flow pattern also depends on (iv) the ratio of the vibrating amplitudes and (v) the phase shift. A weakly nonlinear analysis of nearly resonant forced vibrations (with $\Omega \sim 1$ ) and of the resulting steady streaming flow was given by Nicolás \& Vega (1996) (see also Mollot et al. 1993 for related experiments), while the steady streaming due to non-resonant vibrations was considered by Nicolás et al. (1997). A brief summary of these theoretical results is now given for convenience. If either $(a) \Omega$ is close to a natural inviscid frequency, or $(b)$ only one disk is vibrated, or (c) both disks are vibrated either in phase or in counterphase, then the forcing tangential stress near the interface vanishes to the leading order and the steady streaming flow is driven mainly by the forcing tangential velocity near the disks, and exhibits several interesting patterns. But it is not convenient to counterbalance thermocapillary flows because the price for any eventual comterbalancing effect is to introduce large tangential velocities near the disks. If instead $\Omega$ is not close to resonance and both disks are vibrated with the same frequency and a phase shift different from 0 and $\pi$, then the forcing tangential stress near the free surface is non-zero and counterbalancing is feasible. But undesirable tangential velocities near the disks are still present at leading order if $\Omega \sim 1$. Fortunately, the forcing tangential stress dominates the forcing velocities as $\Omega$ increases. Then by taking $\Omega$ as large as possible (but still small compared to $C^{-1}$ ) and the phase shift equal to $\pi / 2$, a qualitatively antisymmetric steady streaming flow is obtained that annihilates, with surprising accuracy, some thermocapillary flows (Nicolás et al. 1997), namely those resulting from fixing the (different) temperatures of the disks when the interface is thermally insulated provided that the Prandtl number is appropriately small. Nevertheless, this steady streaming flow cannot be symmetric and thus is not the appropriate one to counterbalance the (symmetric) thermocapillary flows associated with the float-zone technique. But this limitation disappears in the next limit, whose analysis is the main object of this paper.

(B) If $\Omega \sim C^{-1}$ or, more generally, if $1 \ll \Omega \ll C^{-3}$, then our analysis below shows that the oscillating and mean flows exhibit the following main features. The oscillating velocity field is a superposition of two sub-fields that are nearly inviscid except in oscillatory boundary layers. A first oscillating sub-field exhibits a wavelength of the order of the size of the liquid bridge, and the second one consists of two counterpropagating capillary wavetrains, with a very small wavelength, which affect only a vicinity of the interface. Those wavetrains are reflected at and forced by the vibrating disks; their amplitudes decay by viscous effects in a length of the order of $(\Omega C)^{-1}$ and may be seen as a linear superposition of a large number of capillary eigenmodes that are excited at the same time. The oscillatory boundary layers, near the disks and the interface, produce steady forcing terms, as in limit (A) above. But now, (a) the tangential stress near the interface due to the counterpropagating capillary wavetrains dominates the remaining steady forcing terms and $(b)$ the distribution of this steady forcing stress along the interface may be controlled by playing with the vibrating frequencies and amplitudes of the disks, and with the phase shift. The 
result is that now (c) several symmetric, antisymmetric and more general streaming flow patterns are obtained by appropriately selecting the vibrating parameters, and (d) the undesirable steady forcing velocities near the disks are essentially absent. We must point out here that the oscillating velocity fields forced by high-frequency axial vibrations in capillary bridges are of independent interest (response of the bridge to high-frequency $g$-jitter) and have not been considered in the literature; thus, a quantitatively precise analysis of the oscillating fields is also included in this paper. Notice that there is an overlap between this limit and limit (A) above (for, if $1 \ll \Omega \ll C^{-1}$ then both limits apply) and thus some of the conclusions by Nicolás et al. (1997) will be obtained again in this paper from a somewhat different point of view.

(C) If $\Omega \sim C^{-3}$ or, more generally, if $\Omega \gg C^{-1}$ then the viscous damping length of the counterpropagating wavetrains is very small, of the order of $(\Omega C)^{-1}$. As a consequence the outgoing wavetrain forced by the high-frequency vibration of each disk survives only a small distance, and the associated steady forcing terms have a little effect on the capillary bridge. Instead, the steady forcing terms due to the other vibrating field, with a larger wavelength, come into play. But this limit is less interesting in liquid bridges (from the practical point of view) than limits (A) and (B) above because, if $C \leqslant 10^{-2}$ and the capillary time $\left(\rho R^{3} / \sigma\right)^{1 / 2}$ is not much larger than $1 \mathrm{~s}$, as is usually the case in practice, then $\Omega \sim C^{-3} \sim 10^{6}$ which means that the forcing frequency is unrealistically large $\left(\sim 10^{6} / 2 \pi \mathrm{Hz}\right)$.

The paper is organized as follows. The mathematical problem is formulated in $\$ 2$. where thermal effects are completely ignored, in order to focus on the structure of the steady streaming flow due to vibration. An asymptotic model giving the steady streaming in the bulk (i.e. outside thin layers near the disks and the interface) will be obtained in $\$ 3$, where a careful analysis of the leading oscillating flow will be also presented (in $\$ 3.1$ ); the validity limits of the asymptotic model will be considered in \$3.3. The above-mentioned counterpropagating capillary wavetrains will be discussed in $\$ 4$. The asymptotic model will be numerically integrated in $\$ 5$ to analyse the several, qualitatively different, streaming flow patterns that are obtained for varying values of the vibrating parameters. Finally, some concluding remarks will be made in $\$ 6$, with some emphasis on the expected ability of the steady streaming flows obtained to counterbalance thermocapillary convection.

\section{Formulation}

Let us consider a liquid bridge of length $L$, held by surface tension between two parallel planar circular coaxial disks of equal radii $R$, that are vibrating in the axial direction with the same frequency and small (compared to $L$ ) amplitudes. The volume of the liquid equals that of the space in the cylinder bounded by the disks and the free surface is assumed to be anchored at the borders of the disks. In addition we neglect gravity and assume that the density $\rho$, viscosity $\mu$ and surface tension $\sigma$ are uniform and constant, and that $\rho$ and $\mu$ are large compared to the corresponding properties of the surrounding gas, which may be thus ignored in the stress balances at the free surface. If we use $R$ and $\left(\rho R^{3} / \sigma\right)^{1 / 2}$ (the capillary time) to non-dimensionalize lengths and time, then the governing equations (continuity and momentum conservation) and boundary conditions (non-slip and anchorage of the free surface at the disks, smoothness of the velocity and pressure fields at the axis of symmetry, and kinematic compatibility and tangential and normal stress balances at 
the free surface) are

$$
\begin{aligned}
& u_{r}+r^{-1} u+w_{z}=0, \\
& u_{t}+w\left(u_{z}-w_{r}\right)=-q_{r}+C\left(u_{r r}+r^{-1} u_{r}-r^{-2} u+u_{z z}\right), \\
& w_{t}+u\left(w_{r}-u_{z}\right)=-q_{z}+C\left(w_{r r}+r^{-1} w_{r}+w_{z z}\right), \\
& u=0, \quad w=h_{ \pm}^{\prime}(t), \quad f=1 \quad \text { at } z= \pm A+h_{ \pm}(t), \\
& u=w_{r}=q_{r}=0 \text { at } r=0, \\
& u=f_{r}+f_{z} w \text { at } r=f, \\
& \left(w_{r}+u_{z}\right)\left(1-f_{z}^{2}\right)+2\left(u_{r}-w_{z}\right) f_{z}=0 \text { at } r^{r}=f, \\
& q-\frac{u^{2}+w^{2}}{2}+\frac{f f_{z z}-1-f_{z}^{2}}{f\left(1+f_{z}^{2}\right)^{3 / 2}}=2 C \frac{u_{r}-\left(w_{r}+u_{z}\right) f_{z}+w_{z} f_{z}^{2}}{1+f_{z}^{2}} \text { at } r=f,
\end{aligned}
$$

with appropriate initial conditions (that are assumed to be axisymmetric). Here, a cylindrical coordinate system is used with associated unit vectors $e_{\mathrm{r}}, e_{\phi}$ and $e_{z}$; $u e_{r}+w e_{z}$ is the velocity, $q$ is the stagnation pressure and the shape of the interface is given by $r=f(z, t)$.

The functions $h_{ \pm}$, giving the instantaneous position of the disks are assumed to be given by

$$
h_{ \pm}(t)=\varepsilon \beta_{ \pm} \mathrm{e}^{\mathrm{i} \Omega t}+\text { c.c. },
$$

where $\varepsilon>0$ is an appropriately small real parameter, the complex amplitudes $\beta_{ \pm}$are of order unity, the frequency $\Omega$ is large, and c.c. stands for the complex conjugate, as is usual. Notice also that the total volume of the liquid bridge is conserved (as a consequence of (2.1), (2.4)-(2.6)) and, according to the assumption above, equals $2 \pi A$, i.e.

$$
\int_{-A+h_{-}}^{A+h_{+}} f(z, t)^{2} \mathrm{~d} z=2 A .
$$

In addition to the forcing vibrating parameters defined above, the problem depends on the slenderness of the bridge, $A=L / 2 R$ and the modified or capillary Reynolds number, $C^{-1}=(\rho \sigma R)^{1 / 2} / \mu$. We shall consider the distinguished limit

$$
\Omega^{-1} \sim C \ll 1, \quad \varepsilon^{2} \Omega^{7 / 3} \sim C .
$$

These conditions imply that the non-dimensional viscous dissipation length $(1 /(\Omega C))$ and the effective Reynolds number associated with the streaming flow $\left(\varepsilon^{2} \Omega^{7 / 3} / C\right)$ are of order unity. But our results below apply provided that

$$
\varepsilon^{2} \Omega^{1 / 3} \ll C \text { and } \varepsilon^{2} \Omega^{17 / 6} C^{1 / 2} \ll 1,
$$

as will be explained in $\$ 3.5$, where it will be also seen that if the steady streaming flow is symmetric (on the plane $z=0$ ) then the second restriction in $(2.12 a)$ may be relaxed to

$$
\varepsilon \Omega \ll 1 .
$$

\section{Derivation of the asymptotic model}

The derivation of the asymptotic model giving the streaming flow in the bulk requires first an analysis of the fairly complex structure of the leading oscillating flow. Six distinguished regions must be considered in the liquid bridge (see figure 1): (a) the bulk, that occupies most of the liquid bridge; (b) a nearly inviscid interface 


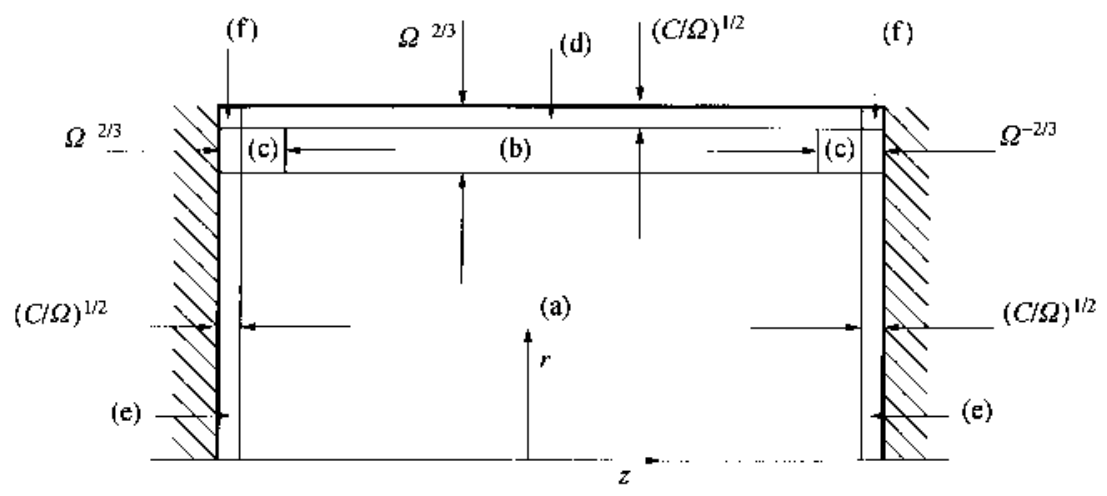

FIGURE 1. Sketch of the six distinguished regions in the liquid bridge.

sub-layer; (c) two nearly inviscid corner regions; (d) a viscous interface boundary layer; (e) two viscous Stokes boundary layers; and (f) two viscous corner boundary regions. The oscillatory velocity field exhibits a wavelength of order unity in region (a), and consists of a pair of counterpropagating capillary wavetrains, with a wavenumber $k=\Omega^{2 / 3} \gg 1$, in region (b) (whose thickness is of the order of $k^{-1} \ll 1$ ). Those wavetrains are reflected and forced in regions (c), whose characteristic size is also of the order of $k^{-1}$.

The oscillating flow in regions (a), (b) and (c) is considered in $\$ 3.1$, and the streaming flow in region (b) is analysed in $\$ 3.2$, where the forcing tangential stress (at the internal edge of this region) driving the streaming flow in the bulk is obtained. Let us anticipate here that this is the main mechanism that produces the streaming flow and essentially comes from the steady tangential stress at the internal edge of the viscous boundary layer (d), first considered by Longuet-Higgins (1953); the steady forcing tangential velocity at the edge of the oscillatory boundary layers (e) (first considered by Schlichting 1932) is a higher-order effect in the limit considered in this paper. But, for the sake of brevity, the fairly involved analysis of the oscillatory boundary layers (d) and (e) is omitted (a summary is given in Appendix B).

\subsection{The oscillating flow in regions (a), (b) and (c)}

In order to facilitate the reading of this section, some lengthy expressions have been relegated to Appendix A.

The oscillatory solution in region (a) may be written as

$$
\left.\begin{array}{c}
u=\varepsilon \Omega\left(\mathrm{ie}^{\mathrm{i} \Omega t} U(r, z)+\text { c.c. }+ \text { HOT }\right), \quad w=\varepsilon \Omega\left(\mathrm{ie}^{\mathrm{i} \Omega t} W(r, z)+\text { c.c. }+ \text { HOT }\right), \\
q-1=\varepsilon \Omega^{2}\left(\mathrm{e}^{\mathrm{i} \Omega t} Q(r, z)+\text { c.c. }+ \text { HOT }\right),
\end{array}\right\}
$$

where HOT stands for higher-order terms, of the order of $\varepsilon^{2}$, and $U, W$ and $Q$ are given by

$$
\begin{gathered}
U_{r}+r^{-1} U+W_{z}=U-Q_{r}=W-Q_{z}=0 \quad \text { in } 0<r<1, \quad-A<z<A, \\
W=\beta_{ \pm} \quad \text { at } z= \pm A, \quad U=W_{r}=0 \quad \text { at } r=0, \quad Q=0 \quad \text { at } r=1 .
\end{gathered}
$$

Equations (3.2) are obtained upon substitution of (3.1) into (2.1)-(2.3), and in (3.3)

+ A detailed analysis of these boundary layers may be obtained from either the Editor or the authors upon request. 
we are using the matching conditions with regions (b) and (c). The unique solution of the linear problem (3.2)-(3.3) is given in the Appendix A. Notice that the solution exhibits a logarithmic singularity near the edge of the disks, $r=1, z= \pm A$, which will play a role below.

The inviscid sub-layer (b) is located near the interface, and has a thickness of the order of $k^{-1}$, where $k>0$ is the wavenumber of the inviscid capillary waves, that satisfy the dispersion relation

$$
\Omega^{2}=k^{3} \text {. }
$$

In addition to the slow axial variable $z$, the fast stretched variables

$$
\xi=k(1-r) \text { and } \eta=k z
$$

are used, and the oscillatory velocity and pressure fields in this region are written as

$$
\left.\begin{array}{rl}
u & =\mathrm{i} \Omega \varepsilon \mathrm{e}^{\mathrm{i} \Omega t}\left[U(1-\xi / k, z)+u_{1}+k^{-1} u_{2}+\mathrm{HOT}\right]+\text { c.c., } \\
w & =\mathrm{i} \Omega \varepsilon \mathrm{e}^{\mathrm{i} \Omega t}\left[W(1-\xi / k, z)+w_{1}+k^{-1} w_{2}+\mathrm{HOT}\right]+\text { c.c., } \\
q-1 & =\Omega^{2} \varepsilon \mathrm{e}^{\mathrm{i} \Omega t}\left[Q(1-\xi / k, z)+k^{-1}\left(q_{1}+k^{-1} q_{2}\right)+\mathrm{HOT}\right]+\text { c.c., }
\end{array}\right\}
$$

where $U, W$ and $Q$ are as defined above and given by (A 1)-(A 4), $u_{j}, w_{j}$, and $q_{j}$ (for $j=1$ and 2 ) depend on $z, \xi$ and $\eta$, and HOT stands for higher-order terms, small compared to those displayed. The shape of the interface, $f$, will also appear in the boundary conditions. For convenience, $f$ is expanded as

$$
f-1=\varepsilon \mathrm{e}^{\mathrm{i} 2 t}\left[U(1, z)+f_{1}+k^{-1} f_{2}+\mathrm{HOT}\right]+\text { c.c., }
$$

where $U(1, z)$ is obtained from (A 1)-(A 4), and $f_{1}$ and $f_{2}$ may depend on $z$ and $\eta$. The first-order problem giving $u_{1}, w_{1}, q_{1}$ and $f_{1}$ is found to be

$$
\begin{gathered}
-u_{1 \xi}+w_{1 \eta}=u_{1}+q_{1 \xi}=w_{1}-q_{1 \eta}=0, \\
u_{1}=w_{1}=q_{1}=0 \quad \text { at } \xi=\infty, \quad u_{1}-f_{1}=q_{1}+f_{1 \eta \eta}=0 \quad \text { at } \xi=0 .
\end{gathered}
$$

The bounded solutions of the problem $(3.8)-(3.9)$ are

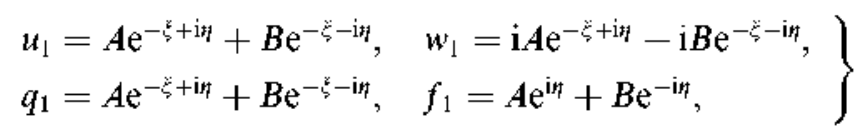

where the complex scalars $A$ and $B$ depend on the slow axial variable $z$ and remain undetermined at this stage. Similarly, the second-order problem giving $t_{2}, w_{2}, q_{2}$ and $f_{z}$ is written and solved in Appendix A, where it is seen that the constants $A$ and $B$ appearing in (3.10) must satisfy the following equations:

$$
3 A_{z}-(4 \omega+\mathrm{i} / 2) A=3 B_{z}+(4(\omega+\mathrm{i} / 2) B=0,
$$

which are obtained as solvability conditions. The real parameter $\omega$ is

$$
\omega=\Omega C \sim 1 .
$$

When (3.10) is substituted into (3.6)-(3.7) it is seen that this part of the oscillatory solution corresponds to a pair of counterpropagating wavetrains, with slowly varying complex amplitudes $A$ and $B$, a wavenumber $k \gg 1$ (see (2.11) and (3.4) and a wave-speed (or phase velocity. Witham 1974)) $\Omega / k=k^{1 / 2} \gg 1 ; A$ and $B$ provide the local amplitudes and phases of the wavetrains. Equations (3.11) are the amplitude equations that provide the spatial decay of $|A|$ and $|B|$ due to viscous dissipation, and the spatial variation of the phases of $A$ and $B$ due to a first local correction 
of the wavenumber. In fact, if time derivatives in a slower time scale, $t \sim k^{1 / 2}$, were included in the amplitude equations, then the terms $3 A$ z and $3 B_{z}$ would account for propagation with the group velocity (which is equal to $3 k^{1 / 2} / 2$ in the original unscaled variables); the other terms account for viscous dissipation and a first correction to the wavenumber.

The amplitude equations (3.11) apply in region (b), i.e. whenever $z+A \gg k^{-1}$ and $A-z \gg k^{-1}$. In order to calculate $A$ and $B$ we need two boundary conditions, that will be obtained now from matching conditions between the oscillatory solutions in regions (b) and (c). To this end, we need the asymptotic behaviour, as $z \rightarrow \pm A$, of the oscillatory solution in region (b). For brevity, we only consider the asymptotic behaviour of the shape of the interface, which is given in Appendix A ((A 10)-(A 11)).

Let us now consider the nearly inviscid corner regions (c). These regions are located near the border of the disks, $r=1, z= \pm A$, and their size is of the order of $k^{-1}$, where $k>0$ is as given by (3.4). Both regions exhibit the same asymptotic structure and could be analysed at the same time. But, for clarity, we shall only describe in detail that region near $r=1, z=A$, in terms of the stretched variables

$$
\xi=k(1-r) \text { and } \hat{\eta}=k(A-z) \text {. }
$$

The oscillatory solution in this region is written as

$$
\left.\begin{array}{c}
u=\mathrm{i} \Omega \varepsilon \mathrm{e}^{\mathrm{i} \Omega t} \tilde{u}_{1}+\mathrm{ccc}+\mathrm{HOT}, \quad w=\mathrm{i} \Omega \varepsilon \mathrm{e}^{\mathrm{i} \Omega t} \tilde{w}_{1}+\text { c.c. }+ \text { HOT, } \\
-1=\left(\Omega^{2} / k\right) \varepsilon \mathrm{e}^{\mathrm{i} \Omega t} \tilde{q}_{1}+\text { c.c. }+ \text { HOT }, \quad f-1=\varepsilon \mathrm{e}^{\mathrm{i} \Omega t} \tilde{f}_{1}+\text { c.c. }+ \text { HOT, }
\end{array}\right\}
$$

where the functions $\tilde{u}_{1}=\tilde{u}_{1}(\xi, \tilde{\eta}), \tilde{w}_{1}=\tilde{w}_{1}(\xi, \tilde{\eta}), \tilde{q}_{1}=\tilde{q}_{1}(\xi, \tilde{\eta})$ and $\tilde{f}_{1}=\tilde{f}_{1}(\tilde{\eta})$ are given by

$$
\begin{aligned}
& \tilde{u}_{1 \xi}+\tilde{w}_{1 \tilde{\eta}}=\tilde{u}_{1}+\tilde{q}_{1 \xi}=\tilde{w}_{1}+\tilde{q}_{1 \tilde{\eta}}=0 \quad \text { in } 0<\xi<\infty, \quad 0<\tilde{\eta}<\infty, \\
& \tilde{w}_{1}=\beta_{+}, \quad \tilde{f}_{1}=0 \text { at } \tilde{\eta}=0, \quad \tilde{u}_{1}-\tilde{f}_{1}=\tilde{q}_{1}+\tilde{f}_{1}^{\prime \prime}=0 \text { at } \xi=0, \\
& \left|\tilde{f}_{1}\right| \text { diverges at most logarithmically as } \hat{\eta} \rightarrow \infty .
\end{aligned}
$$

Equations (3.15) are obtained upon substitution of (3.13)-(3.14) into (2.1)-(2.3), and in conditions (3.16)-(3.17) we are using matching conditions with regions (a), (b), (d) and (e). This linear problem is solved in Appendix A ((A 12)-(A 13)), where the following boundary condition for the complex amplitudes is found:

$$
A=\mathrm{e}^{-2 \mathrm{iv}} B-\mathrm{ie}^{-\mathrm{iv}}\left(\gamma_{1} \beta_{+}-\gamma_{2} \beta_{-}\right) \text {at } z=A,
$$

upon application of matching conditions between the solution in this region (as $\tilde{\eta} \rightarrow \infty$ ) and that of region (b) (as $z \rightarrow A$ ). Here, the real scalars $v_{,} \gamma_{1}$ and $\gamma_{2}$ are

$$
v=k A-\pi / 3, \quad \gamma_{1}=\sqrt{3}\left[(2 / \pi) \log k-b_{1}+b_{2}-\delta_{1}\right] / 2+\frac{1}{3}, \quad \gamma_{2}=\sqrt{3} \delta_{2} / 2,
$$

where $\delta_{1}, \delta_{2}, b_{1}$ and $b_{2}$ are as defined in (A 11), (A 14) and (A 16). The constants $\gamma_{1}-(2 \sqrt{3} \log k) / \pi$ and $\gamma_{2}$ are plotted vs. the slenderness in figure 2 . A similar analysis of region (c) near $r=1, z=-A$ yields

$$
B=\mathrm{e}^{-2 \mathrm{i} i} \boldsymbol{A}+\mathrm{ie}^{-\mathrm{i} v}\left(\gamma_{1} \beta_{-}-\gamma_{2} \beta_{+}\right) \text {at } z=-\boldsymbol{A} .
$$

The first terms in the right-hand sides of the boundary conditions (3.18) and (3.20) account for pure reflection of the wavetrains in region (c), with a complex reflection coefficient $\mathrm{e}^{-2 \mathrm{i} v}$, whose argument yields a phase shift between the incoming and the outgoing waves. Viscous dissipation in the Stokes boundary layer inside region (c) would give a correction to the reflection coefficient that is seen to be small in the 


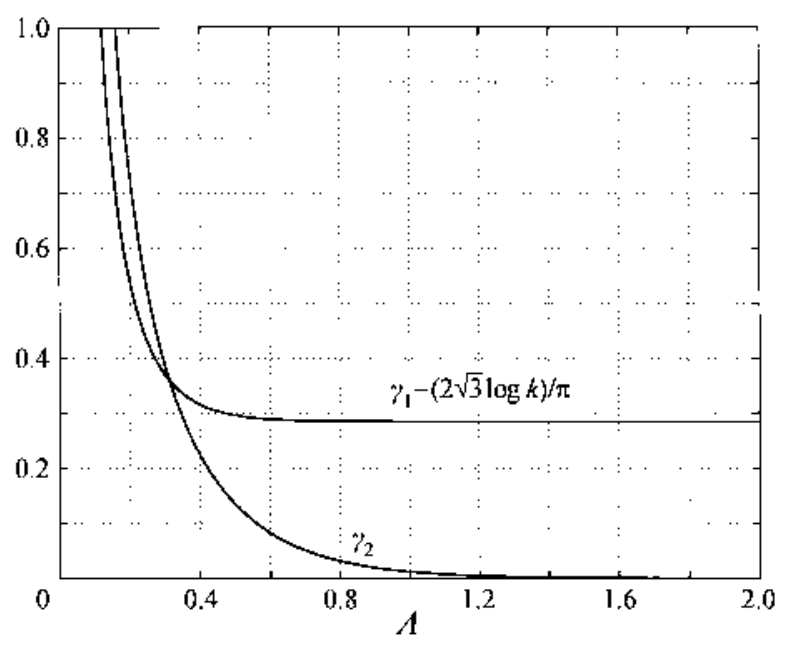

FIGURE 2. The constants $\eta_{1}-(2 \sqrt{3} \log k) / \pi$ and $\gamma_{2}$ (appearing in the boundary conditions (3.33) and (3.35)) in terms of the slenderness $A$.

limit (2.11); thus we neglect this correction which yields a small absorption of the incoming wave in region (c). The second term in the right-hand sides of (3.18) and (3.20) depends linearly on the scaled forcing complex amplitudes $\beta_{+}$and $\beta_{-}$(see (2.9)) and account for forcing in region (c). Two remarks about these forcing terms are now in order.

(i) We have included a two-terms approximation of forcing, namely a leading-order one that is proportional to $\log k$ and a first correction that is of order one; we are instead ignoring terms that are of the order of $k^{-1} \log k$. This has been done in order to obtain a quantitatively good approximation of the complex amplitudes (which would not be obtained if the $O(1)$ term were ignored), as will be checked in $\$ 4$.

(ii) A closer look at the matching conditions (in Appendix A) that led to the boundary conditions (3.18) shows that the leading forcing term, namely that proportional to $\beta_{+} \log k$ at $z=A$, accounts for direct forcing from the solid disk that is vibrated around $z=A$ and has a local nature. The second, $O(1)$-term instead accounts for forcing from the bulk; thus it is not surprising that this term is non-local, namely it does not only depend on the scaled forcing amplitude, $\beta_{+}$, of the disk that is vibrated around $z=A$, but it also depends on the forcing complex amplitude $\beta_{-}$of the other vibrating disk. Notice that since $\left|\gamma_{1} / \gamma_{2}\right| \sim \log k \gg 1$, non-locality is fairly weak; if $A \gtrsim 1$ then $\left|\gamma_{2}\right|$ becomes extremely small (see figure 2) and forcing becomes essentially local.

\subsection{The leading-order steady flow in region (b)}

As in $\$ 3.1$, the fast spatial variables (3.5) are used. The steady part of the solution in this region is written as

$$
\left.\begin{array}{c}
u=\varepsilon^{2} \Omega k u_{1}^{s}+\mathrm{HOT}+\mathrm{NST}, \quad w_{\xi}=\varepsilon^{2} \Omega k h_{1}+\mathrm{HOT}+\mathrm{NST}, \\
q=\varepsilon^{2} \Omega C k^{2} q_{1}^{s}+\mathrm{HOT}+\mathrm{NST},
\end{array}\right\}
$$

where, as above, HOT stands for higher-order terms, and NST stands for non-steady terms. If (3.5) and (3.21) are inserted into (2.1)-(2.3), then the following equations 
and boundary conditions result:

$$
\begin{aligned}
& u_{1 \xi \xi}^{s}-h_{1 \eta}=u_{1 \xi \xi}^{s}+u_{1 \eta \eta}^{s}+q_{1 \xi}^{s}=h_{1 \xi \xi}+h_{1 \eta \eta}-q_{1 \xi \eta}^{s}=0, \\
& u_{1}^{s}=-\left[\mathrm{i} \bar{U}(1, z)\left(A \mathrm{e}^{\mathrm{i} \eta}+B \mathrm{e}^{-\mathrm{i} \eta}\right)+\text { c.c. }\right] \text { at } \xi=0, \\
& h_{1}=-\left[\bar{U}(1, z)\left(A \mathrm{e}^{\mathrm{i} \eta}-B \mathrm{e}^{-\mathrm{i} \eta}\right)+\text { c.c. }\right]-8\left(|B|^{2}-|A|^{2}\right) \text { at } \xi=0, \\
& u_{1 \xi}=h_{1 \xi}=0 \text { at } \xi=\infty,
\end{aligned}
$$

where (in (3.23)-(3.25)) we are using matching conditions with regions (a) and (d) (see (B 3) and (B 4) in Appendix B). Integration of (3.22)-(3.25) yields

$$
\left.\begin{array}{c}
u_{1}^{s}=-\left[\mathrm{i} \bar{U}(1, z)\left(A \mathrm{e}^{\mathrm{i} \eta}+B \mathrm{e}^{-\mathrm{i} \eta}\right) \mathrm{e}^{-\xi}+\text { c.c. }\right], \\
h_{1}=-\left[\bar{U}(1, z)\left(A \mathrm{e}^{\mathrm{i} i t}-B \mathrm{e}^{-\mathrm{i} \eta}\right) \mathrm{e}^{-\xi}+\text { c.c. }\right]-8\left(|B|^{2}-|A|^{2}\right) .
\end{array}\right\}
$$

These expressions for $u_{1}^{s}$ and $h_{1}$ show that the steady part of the velocity field in this region consists of two parts. A first one corresponds to a stationary hydrodynamic wave that exhibits a fast spatial oscillation in the axial direction, with a wavenumber $k \gg 1$ that results from the spatially oscillatory terms in the boundary conditions (3.23)-(3.24) and decays exponentially as $\zeta \rightarrow \infty$ (i.e. this wave does not penetrate into the bulk). A second part (namely the last term in the expression for $h_{1}$ ) exhibits an axial wavelength of order unity and does penetrate into the bulk; in fact, this part provides the main driving mechanism for the steady flow in the bulk.

Now, we only need to take into account (3.5), (3.21) and (3.26), and apply matching conditions with the solution in the bulk to obtain the following boundary conditions near the interface:

$$
u=o\left(\varepsilon^{2} \Omega k\right)+\mathrm{NST}, \quad w_{r}=8 \varepsilon^{2} \Omega k^{2}\left(|B|^{2}-|A|^{2}\right)+\text { HOT + NST, }
$$

at $r=1$ (for the solution in the bulk).

\subsection{The asymptotic model}

Let us now collect the results above concerning the amplitudes, $A$ and $B$, of the counterpropagating wavetrains in region (b) and the steady flow in the bulk. The amplitudes $A$ and $B$ are given by equations (3.11), with boundary conditions (3.18) and (3.20), where the real constants $\omega, v, \gamma_{1}$ and $\gamma_{2}$ are given by (3.12) and (3.19), and $\beta_{+}$and $\beta_{-}$are the scaled complex amplitudes associated with disk vibration (see (2.4) and (2.9)). Similarly, the leading steady parts of the velocity and pressure fields in the bulk are given by (see (2.1)-(2.3))

$$
\begin{aligned}
& u_{r}^{s}+r^{-1} u^{s}+w_{z}^{s}=0, \\
& w^{s}\left(u_{z}^{s}-w_{r}^{s}\right)=-q_{r}^{s}+C\left(u_{r r}^{s}+r^{-1} u_{r}^{s}-r^{-2} u^{s}+u_{z z}^{s}\right), \\
& u^{s}\left(w_{r}^{s}-u_{z}^{s}\right)=-q_{z}^{s}+C\left(w_{r r}^{s}+r^{-1} w_{r}^{s}+w_{z z}^{s}\right) .
\end{aligned}
$$

Notice that we are neglecting steady terms resulting from products of oscillatory terms in the left-hand sides of (3.29)-(3.30); that assumption will be checked below. The boundary conditions are

$$
\begin{array}{rll}
u^{s}=w^{s}=0 \quad \text { at } z= \pm A, \quad u^{s}=w_{r}^{s}=q_{r}^{s}=0 & \text { at } r=0 \\
u^{s}=w_{r}^{s}+8 \varepsilon^{2} \Omega k^{2}\left(|A|^{2}-|B|^{2}\right)=0 & \text { at } r=1,
\end{array}
$$

as obtained from matching conditions with the steady-state solution in regions (b) and (e) (see (3.27), (B 6) and (B 7)).

Now the steady streaming flow in the bulk is governed by (3.28)-(3.32), and has an 
effective Reynolds number

$$
R e=\left.8 \varepsilon^{2} \Omega k^{2}|| A\right|^{2}-|B|^{2} \mid / C .
$$

If the effective Reynolds number $R e$ is large, the steady streaming flow exhibits viscous boundary layers near the disks and the interface (at least); in the latter we have

$$
u^{s} \sim C R \mathrm{e}^{1 / 3}, \quad w^{s} \sim C R \mathrm{e}^{2 / 3}, \quad l_{c} \sim R \mathrm{e}^{-1 / 3},
$$

where $l_{c}$ is the characteristic thickness of the layer; outside the viscous boundary layers we have

$$
u^{s} \sim w^{s} \sim C R \mathrm{e}^{2 / 3} .
$$

If instead $R e$ is not large then viscous boundary layers are absent and we have $u^{s} \sim w^{s} \sim C R e$ everywhere; notice that (3.34a) or (3.34b) also hold if $R e \sim 1$ is not small (they do not hold if $R e \ll 1$, but we shall not consider small values of $R e$ below).

Now we have a look at the steps leading to (3.11), (3.18), (3.20) and (3.28)-(3.32) to obtain the validity limits of this model:

(a) The characteristic thickness $(C / \Omega)^{1 / 2}$ of the oscillatory viscous boundary layer near the interface must be small compared to the thickness $\left(k^{-1}\right)$ of the inviscid layer near the interface, and the latter must be small as compared to 1 for our analysis above to be valid. When taking into account the dispersion relation (3.4), those conditions put two restrictions on the forcing frequency

$$
1 \ll \Omega \ll C^{-3} .
$$

(b) The leading nonlinear terms that have been neglected in the amplitude equations (3.11) are given by $i \varepsilon^{2} \Omega^{2}\left(\alpha_{1}|A|^{2}+\alpha_{2}|B|^{2}\right) A+\mathrm{i} k^{1 / 2} \alpha_{3} w^{s}(1, z) A$ and $-\mathrm{i} \varepsilon^{2} \Omega^{2}\left(\alpha_{1}|B|^{2}+\right.$ $\left.\alpha_{2}|A|^{2}\right) B+\mathrm{i} k^{1 / 2} \alpha_{3} w^{s}(1, z) B$ in the first and second equations respectively, where $\alpha_{1}, \alpha_{2}$ and $\alpha_{3}$ are real constants. These terms may be neglected provided that

$$
\Omega^{1 / 3} \mathrm{Re}^{2 / 3} \mathrm{C} \ll 1 \quad \text { or } \varepsilon^{2} \Omega^{17 / 6} \mathrm{C}^{1 / 2} \ll 1 \text {, }
$$

as readily obtained when taking into account (3.4), (3.33) and (3.34a). If, in addition, the steady flow is symmetric then $w^{s}(1, z)$ is an odd function and the associated nonlinear terms may be eliminated by adding the quantity $\mathrm{i} k^{1 / 2} \alpha_{3} \int_{0}^{z} w^{\mathrm{s}}(1, z) \mathrm{d} z / 3$ to the phases of $A$ and $B$ (notice that this change does not affect the forcing term in the boundary condition (3.32)). In that case, condition (3.36a) may be relaxed to

$$
C R e \ll \Omega^{1 / 3} \text { or } \varepsilon \Omega \ll 1 \text {. }
$$

In addition, we have neglected dispersion which could have been taken into account by adding the new terms $-(3 \mathrm{i} / 2 k) \boldsymbol{A}_{\bar{z} \bar{z}}$ and $(3 \mathrm{i} / 2 k) B_{\bar{z}}$ to the left-hand sides of (3.11) (then a second pair of boundary conditions should be added at $z= \pm A$, which would be obtained from higher-order matching conditions with the solution in region (c)). These new terms are smaller than those considered unless $k^{-1}\left|A_{z z}\right| \sim|A|$ and/or $k^{-1}\left|B_{z}\right| \sim|B|$, that is, unless intermediate scales, with a wavelength of the order of $k^{-1 / 2}$ (that is between the basic wavelength $k^{-1}$ of the capillary wavetrains and the size of the bridge) come into play. The analysis of these intermediate scales is a subtle matter that requires inertia to be considered also (which has been neglected in (3.11)); see Martel \& Vega (1996) for an analysis of these scales in a related problem. The analysis by Martel \& Vega (1996) suggests that intermediate scales can be ignored unless nonlinearity is present and thus our assumption on neglecting dispersion seems to be justified. 
(c) If the effective Reynolds number $R e$ is large, then the thickness of the steady viscous boundary layers associated with the steady streaming flow must be large compared to $k^{-1}$ (the thickness of region (b), which was used to obtain the boundary conditions (3.32)). That condition holds provided that $l_{c} \sim R \mathrm{e}^{-1 / 3} \gg k^{-1}$, that is

$$
R e \ll k^{3}=\Omega^{2} \quad \text { or } \quad \varepsilon^{2} \ll C \Omega^{-1 / 3} .
$$

Similarly, the viscous boundary layers near the disks must be also much thicker than $(C / \Omega)^{1 / 2}$ (that is, the thickness of region (e), which was used to derive the boundary conditions (3.31) at $z= \pm A$ ). But this condition puts a condition on the effective Reynolds number that is less stringent than those imposed above.

(d) Steady terms resulting from products of oscillatory terms have been neglected in momentum conservation equations, (3.29)-(3.30). These neglected terms are given by

$$
\begin{aligned}
& w^{a}\left(u_{z}^{s}-w_{r}^{s}\right)+\left[i \Omega U \bar{W}\left(u_{z}^{s}-w_{r}^{s}\right)+\text { c.c. }\right]_{r}, \\
& u^{a}\left(w_{r}^{s}-u_{z}^{s}\right)+\left[i \Omega U \bar{W}\left(u_{z}^{s}-w_{r}^{s}\right)+\text { c.c. }\right]_{z},
\end{aligned}
$$

respectively, where

$$
u^{n}=-\varepsilon^{2} \Omega\left[\mathrm{i}(\bar{U} W)_{z}+\text { c.c. }\right], \quad w^{a}=\varepsilon^{2} \Omega \mathrm{ir}^{-1}(r \bar{U} W)_{r}+\text { c.c. }
$$

Notice that the second terms in (3.38)-(3.39) derive from a potential and thus may be eliminated by a redefinition of pressure. Then the neglected terms are small compared to those displayed provided that $t^{n}$ and $w^{n}$ may be neglected compared to $u^{s}$ and $w^{s}$, and according to our discussion above on the estimates $(3.34 b)$, this condition holds provided that

$$
\varepsilon^{2} \Omega \ll C R \mathrm{e}^{2 / 3} \text { or } \varepsilon^{2} \ll C \Omega^{5 / 3} .
$$

Notice that we have also neglected steady terms resulting from products of oscillating terms in region (b) (see \$3.2); but a similar analysis to that above shows that those terms may be in fact neglected provided that our restrictions already imposed above hold.

(e) Equations (3.28)-(3.32) have been written in terms of the steady part of the velocity field, $\left(u^{s}, w^{s}\right)$, which has been obtained as a time average of the velocity field. That average does not need to coincide with the drift (or mass-transport) velocity, $\left(u^{d}, w^{d}\right)$, for kinematical reasons (see e.g. Batchelor 1967, where the drift velocity is defined as the velocity associated with the time-averaged trajectories of material elements). In fact, $\left(u^{d}, w^{d}\right)$ (and not $\left(u^{s}, w^{s}\right)$ ) must be used for comparison with experiments because if the liquid bridge is observed with an exposure time that is large compared to the oscillatory period, then the time average of the trajectories of material elements is observed. The components of the drift velocity are given by

$$
u^{d} \simeq u^{s}+u^{a}, \quad w^{d} \simeq w^{s}+w^{a},
$$

where $u^{a}$ and $w^{a}$ are again given by (3.40). Thus if (3.41) holds, then we have $t^{d} \simeq u^{s}$ and $w^{d} \simeq w^{s}$, and (3.28)-(3.32) gives the drift velocity in first approximation.

\section{The counterpropagating capillary wavetrains}

The oscillating flow in the bulk was calculated in $\$ 3.1$ to be as given by (3.1)-(3.3), (A 1)-(A 4) to a first approximation, and exhibits a wavelength of the order of the length of the capillary bridge. This flow is readily seen to be standing (i.e. with a phase 
independent of position) if $\beta_{+} \bar{\beta}_{-}=$real, that is, if either only one disk is vibrated $\left(\beta_{+} \bar{\beta}_{-}=0\right)$ or both disks are vibrated, either in phase $\left(\beta_{+} \bar{\beta}_{-}=\right.$real and positive $)$or in counterphase $\left(\beta_{+} \bar{\beta}_{-}=\right.$real and negative). If, instead, the imaginary part of $\beta_{+} \bar{\beta}_{-}$ is non-zero, then this flow is progressive.

The oscillating flow in the inviscid sub-layer (b) and the shape of the interface $r=f(z, t)$ were calculated in $\$ 3.1$ to be given by $(3.5)-(3.7)$ and (3.10). For brevity we only rewrite here the expression for the shape of the interface,

$$
f-1=\varepsilon \mathrm{e}^{\mathrm{i} \Omega t} F(z)+\text { c.c. }+ \text { HOT, }
$$

where

$$
F(z)=A(z) \mathrm{e}^{\mathrm{i} k z}+B(z) \mathrm{e}^{-\mathrm{i} k z}+\left[\left(\beta_{+}+\beta_{-}\right) U^{o}(1, z)+\left(\beta_{+}-\beta_{-}\right) U^{e}(1, z)\right] / 2,
$$

with $U^{o}(1, z), U^{e}(1, z), A(z)$ and $B(z)$ given by (A 2)-(A 4), (3.11), (3.18) and (3.20). Thus, to the leading order, the oscillating flow in region (b) consists of a mean field and a pair of counterpropagating wavetrains. This flow is always progressive (i.e. its phase depends on position) to a first approximation if $\omega=\Omega C \neq 0$. In the limiting case when $\omega=0$ this flow is seen to be standing if $\beta_{+} \bar{\beta}_{-}=$real and progressive otherwise.

The solution of (3.11), (3.18) and (3.20) is

$$
A=A_{0} \mathrm{e}^{(8 \omega+\mathrm{i}) z / 6}, \quad B=B_{0} \mathrm{e}^{-(8 \omega+\mathrm{i} \xi / / 6},
$$

where the complex constants $A_{0}$ and $B_{0}$ are

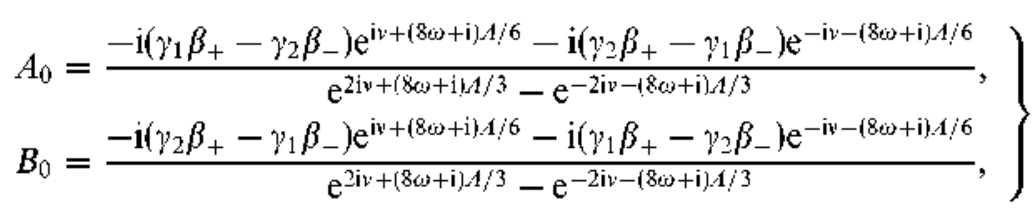

with $\omega, v, \gamma_{1}$ and $\gamma_{2}$ given by (3.12) and (3.19).

For clarity we have considered in $\$ 3$ only the time-independent version of the amplitude equations (3.11). But time derivatives are readily added to obtain

$$
-\left(2 /\left(k^{1 / 2}\right)\right) A_{t}+3 A_{z}-(4 \omega+\mathrm{i} / 2) A=\left(2 /\left(k^{1 / 2}\right)\right) B_{t}+3 B_{z}+(4 \omega+\mathrm{i} / 2) B=0,
$$

where we have just taken into account that the ratio of the coefficients of $A_{z}$ and $B_{z}$ over the coefficients of $A_{t}$ and $B_{t}$ respectively must be equal to the group velocity (see Witham 1974), which equals $\mathrm{d} \Omega / \mathrm{d} k\left(=3 k^{1 / 2} / 2\right)$ and $-\mathrm{d} \Omega / \mathrm{d} k$ respectively (recall that the wavenumber associated with $B$ is negative, according to (3.10)), with $\Omega(k)=k^{3 / 2}$ being the dispersion relation (see (3.4)). Time derivatives will be used below only to calculate the damping rate of the capillary wavetrains.

In order to ascertain that we have obtained a quantitatively good approximation (as $C \rightarrow 0$ and $\Omega \rightarrow \infty$ ) of the oscillatory solution and of the steady forcing term yielding the steady streaming flow in the bulk, some numerical comparisons are now made.

(a) The inviscid eigenfrequencies are readily calculated by first setting $\omega=\beta_{+}=$ $\beta_{-}=0$ in (3.11), (3.18) and $(3.20)$ and then requiring the resulting homogeneous linear problem to have a non-trivial solution or, equivalently, by setting to zero the denominators in (4.4) (and $\omega=0$ ), to obtain $A / 6+v=m \pi / 2$ or, when taking into account (3.4) and (3.19),

$$
\Omega_{m}=[(3 m+2) \pi / 6 A-1 / 6]^{3 / 2}, \quad \text { with } m=\text { integer. }
$$




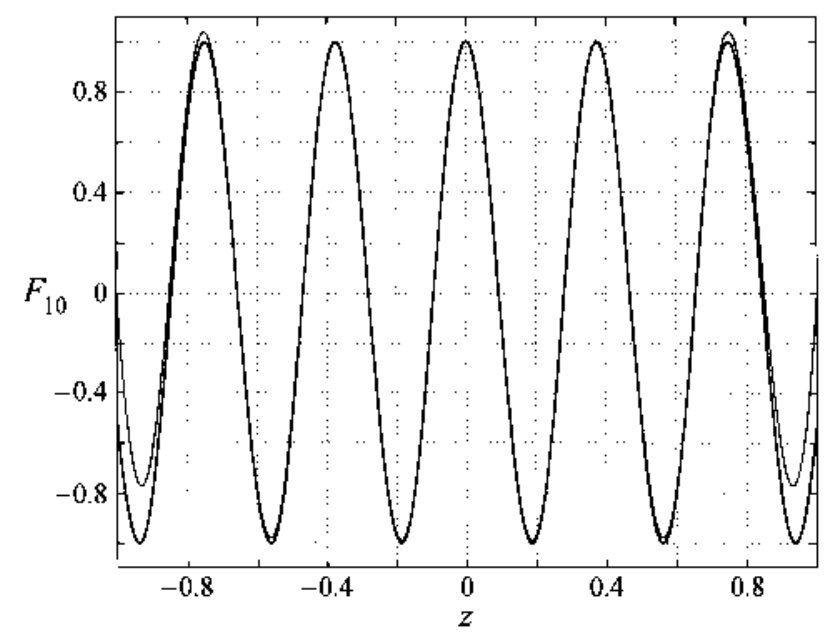

FigurE 3. The tenth eigenfunction $F_{10}$ for $A=1$ : as given by (4.7) (thick line) and as calculated in Sanz (1985) (thin line).

The associated non-trivial solution is given by $A=\mathrm{e}^{\mathrm{i} z / 6}, B=\mathrm{e}^{\mathrm{i}(m \pi-z / 6)}$, up to a common complex factor. When taking into account (4.1)-(4.2) we readily obtain the associated eigenfunction $F_{m}$ (yielding the shape of the interface in the linear, resonant response as $f-1=\varepsilon\left(\mathrm{e}^{\mathrm{i} \Omega_{m} t} F_{m}+\right.$ c.c. $\left.)\right)$ that is (recall that $k=\Omega^{2 / 3}$, see (3.4))

$$
\left.\begin{array}{cl}
F_{m}=\sin \left[\left(\Omega_{m}^{2 / 3}+1 / 6\right) z\right]=\sin [(3 m+2) \pi z / 6 \Lambda] & \text { if } m \text { is odd, } \\
F_{m}=\cos \left[\left(\Omega_{m}^{2 / 3}+1 / 6\right) z\right]=\cos [(3 m+2) \pi z / 6 \Lambda] & \text { if } m \text { is even, }
\end{array}\right\}
$$

up to a constant, complex factor. For illustration, in figure 3 we compare this expression for $F_{m}$ with that calculated by the semi-analytical method due to Sanz (1985) (which is essentially exact) for $A=1$ and $m=10$ (the arbitrary constant has been chosen to be real and such that both expressions coincide at $z=0$ ). Notice that the approximation is quite good except near the disks, $z= \pm A$; the latter was to be expected because if $|z \mp A| \sim \Omega_{m}^{-2 / 3}$ then the solution in region (c) (given by (3.14) and (A 12)-(A 13)) applies instead of (4.7). Also, in table 1 we compare the approximation (4.6) for $\Omega_{m}$ with the essentially exact values calculated by the semi-analytical method (Sanz 1985), for several modes and $A=1$. Notice that (4.6) provides a fairly good approximation even for the first inviscid eigenmode, in spite of the fact that it only needs to apply for high-order modes.

(b) The damping rate at resonance is readily calculated from (4.5) to be

$$
\lambda_{m}=2 \Omega_{m}^{4 / 3} \mathrm{C}
$$

where we have used (3.4) and (3.12), and $\Omega_{m}$ is the associated inviscid eigenfrequency, given by (4.6). In table 1 we compare the approximation (4.8) with the approximation in Higuera, Nicolás \& Vega (1994) (which applies provided that $C \ll 1$ ) for several modes, three values of the capillary Reynolds number, $C^{-1}=10^{2}, 10^{3}$ and $10^{4}$, and $A=1$. Notice that the approximation in this paper is again quite good even for the lower modes.

(c) The linear inviscid non-resonant response of the capillary bridge is given by (4.1)-(4.2), where $U^{o}(1, z), U^{e}(1, z), A(z)$ and $B(z)$ are given by (A 2)-(A 4), (4.3) and (4.4), with $\omega=0$. For illustration we take $A=1$ and $\Omega=104$ (then $v \simeq 21.068$, 


\begin{tabular}{|c|c|c|c|c|c|c|c|c|}
\hline \multirow[b]{2}{*}{$m$} & \multicolumn{2}{|c|}{ Inviscid cigenfreq. } & \multicolumn{2}{|c|}{ Damping ratc $C=0.01$} & \multicolumn{2}{|c|}{ Damping ratc $C=0.001$} & \multicolumn{2}{|c|}{ Damping rate $C=0.000$} \\
\hline & $\Omega_{m}^{\text {exuct }}$ & $\Omega_{m i}^{a p p r o x}$ & $\hat{\lambda}_{m_{1}}^{\text {Lupprax }}$ & $\lambda_{m_{2}}^{\text {apprax }}$ & $\hat{\lambda}_{m i}^{\text {appprax }}$ & $\lambda_{m_{2}}^{\text {approx }}$ & $\lambda_{m i 1}^{\text {approx }}$ & $\lambda_{m_{2}}^{a p p r o x}$ \\
\hline 1 & 3.26 & 3.84 & 0.077 & 0.12 & 0.009 & 0.012 & 0.0014 & 0.012 \\
\hline 2 & 7.52 & 8.06 & 0.265 & 0.162 & 0.0306 & 0.0323 & 0.0044 & 0.0032 \\
\hline 3 & 12.80 & 13.23 & 0.552 & 0.626 & 0.0624 & 0.0625 & 0.0085 & 0.0063 \\
\hline 4 & 18.78 & 19.17 & 0.934 & 1.026 & 0.1028 & 0.1026 & 0.0132 & 0.0103 \\
\hline 5 & 25.47 & 25.81 & 1.416 & 1.526 & 0.1535 & 0.1525 & 0.0191 & 0.0153 \\
\hline 6 & 32.75 & 33.08 & 1.993 & 2.124 & 0.2131 & 0.2124 & 0.0257 & 0.0212 \\
\hline 7 & 40.63 & 40.93 & 2.671 & 2.821 & 0.2830 & 0.2820 & 0.0334 & 0.0232 \\
\hline 8 & 49.02 & 49.31 & 3.445 & 3.616 & 0,3622 & 0.3616 & 0.0418 & 0.0362 \\
\hline 9 & 57.93 & 58.20 & 4.318 & 4.510 & 0.4515 & 0.4510 & 0.0514 & 0.0451 \\
\hline 10 & 67.31 & 67.56 & 5.290 & 5.504 & 0.5503 & 0.5504 & 0.0618 & 0.0550 \\
\hline 15 & 120.63 & 120.84 & 11.623 & 11.948 & 1.196 & 1.196 & 0.1286 & 0.1195 \\
\hline 20 & 183.36 & 183.54 & 20.432 & 20.861 & 2,080 & 2.086 & 0.2197 & 0.2086 \\
\hline 25 & 254.25 & 254.41 & 31.707 & 32.241 & 3.215 & 3.224 & 0.3354 & 0.3224 \\
\hline 30 & 332.45 & 332.60 & 45.440 & 46.088 & 4.595 & 4.609 & 0.4756 & 0.4609 \\
\hline
\end{tabular}

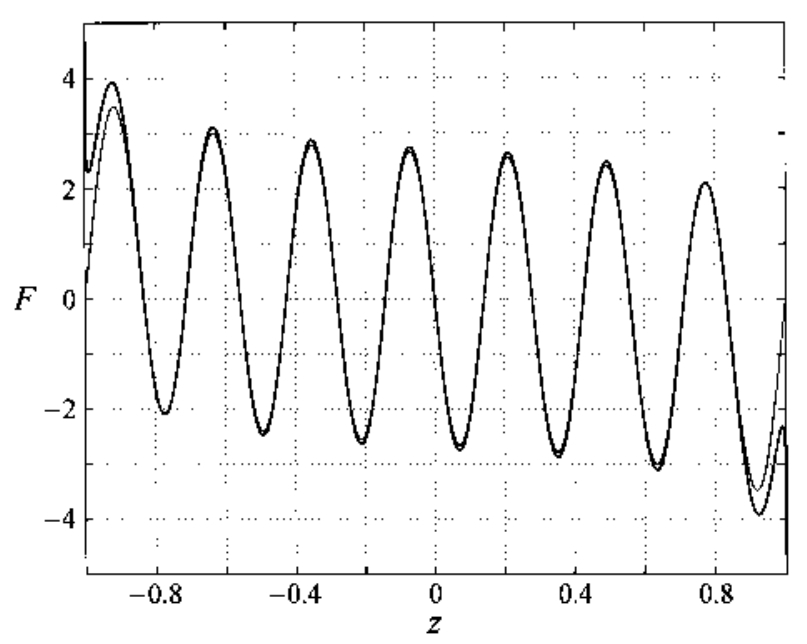

Figure 4. The function $F$ for $A=1, \Omega=104$ and $\beta_{+}=\beta=1$ : as given by (4.2) (thick line) and as calculated in Nicolás et al. (1997) (thin line).

$\gamma_{1} \simeq 1.9912$ and $\gamma_{2} \simeq 0.01175$, as calculated from (A 21)). In figure 4 we compare the function $F$ associated with the linear response of the bridge, in the particular case when $\beta_{+}=\beta_{-}=1$ (then $F$ is real), as calculated above (thick line) and as calculated by means of the approximation by Nicolás et al. (1997) (thin line), which is essentially exact. Notice that (as in figure 3 and for the same reason) the approximation for $F$ in figure 4 is quite good except near the disks, $z= \pm A$.

From the quantitative comparisons above (and others not presented here for brevity) we conclude that our approximation above to the oscillatory solution is quite good provided that $\Omega \geqslant 100$ and $C \leqslant 0.01$. 
Finally, notice that if

$$
\beta_{+} / \beta_{-}=\left[\gamma_{1}+\gamma_{2} \mathrm{e}^{2 \mathrm{iv}+(8 \omega+\mathrm{i}) / / 3}\right] /\left[\gamma_{1} \mathrm{e}^{2 \mathrm{i} w+(8 \omega+\mathrm{i}) / 4 / 3}+\gamma_{2}\right]
$$

then $A_{0}=0$ and one of the capillary wavetrains is absent (see (4.3)-(4.4)). That value of the ratio of the vibrating amplitudes $\left(\left|\beta_{+} / \beta_{-}\right|\right)$and the phase shift (phase of $\beta_{+} / \beta_{-}$) is precisely such that there is a perfect interference between the reflected and forced wavetrains at the disk near $z=A$; then the outgoing wavetrain from that disk disappears. Similarly, the quantity $\left|A_{0}\right| /\left|B_{0}\right|$ (that measures the relative strength of the counterpropagating wavetrains) may be continuously varied from zero to infinity by appropriately chosing (in a non-unique way) the modulus and phase of $\beta_{+} / \beta_{-}$. In particular, if $\beta_{+}= \pm \beta_{-}$(i.e. if both disks are vibrated either in phase or in counterphase, with the same amplitude) then

$$
\left|A_{0}\right|^{2}=\left|B_{0}\right|^{2}=\left(\gamma_{1} \mp \gamma_{2}\right)^{2}\left|\beta_{+}\right|^{2} / 2[\cosh (8 \omega \Lambda / 3) \pm \cos (2 v+\Lambda / 3)] .
$$

If $\beta_{-}=0$, then

$$
\left.\begin{array}{rl}
\left|A_{0}\right|^{2} & =\frac{\gamma_{1}^{2} \exp (8 \omega A / 3)+\gamma_{2}^{2} \exp (-8 \omega A / 3)+2 \gamma_{1} \gamma_{2} \cos (2 v+A / 3)}{\cosh (16 \omega \Lambda / 3)-\cos (4 v+2 A / 3)} \frac{\left|\beta_{+}\right|^{2}}{2}, \\
\left|B_{0}\right|^{2} & =\frac{\gamma_{2}^{2} \exp (8 \omega A / 3)+\gamma_{1}^{2} \exp (-8 \omega A / 3)+2 \gamma_{1} \gamma_{2} \cos (2 v+A / 3)}{\cosh (16 \omega \lambda / 3)-\cos (4 v+2 A / 3)} \frac{\left|\beta_{+}\right|^{2}}{2},
\end{array}\right\}
$$

while if $\beta_{+}=0$ then (4.10) stand after substituting $A_{0}$ by $B_{0}$, and $\beta_{+}$by $\beta_{-}$.

In the inviscid limit, when $\omega=0$, the following equation (that will be needed in \$5) holds:

$$
\left|\boldsymbol{A}_{0}\right|^{2}-\left|B_{0}\right|^{2}=\left(\gamma_{1}^{2}-\gamma_{2}^{2}\right)\left[\left(\bar{\beta}_{+} \beta_{-}-\beta_{+} \bar{\beta}_{-}\right) \mathrm{e}^{2 \mathrm{v} v+\mathrm{i} \Lambda / 3}+\text { c.c. }\right] / 4 \sin ^{2}(2 v+\Lambda / 3) .
$$

Notice that $\left|A_{0}\right|^{2}-\left|B_{0}\right|^{2} \neq 0$ if and only if the imaginary part of $\beta_{+} \bar{\beta}_{-}$is non-zero or, according to our remark at the beginning of this section, if and only if the oscillating flow is progressive to a first approximation; in this case, the sign of $\left|A_{0}\right|^{2}-\left|B_{0}\right|^{2}$ may be controlled through the sign of the phase shift between the forcing vibration of the disks, that is, the phase of $\beta_{+} \bar{\beta}_{-}$.

\section{The steady streaming flow in the bulk}

Let us now consider the steady streaming flow driven by vibration of the disks. As seen in $\$ 3.3$, in a first approximation this flow is governed in the bulk by (3.28)(3.32), with the complex amplitudes $A$ and $B$ as given by (3.11), (3.18) and (3.20). For convenience we re-scale the velocity components and pressure (that is now used instead of the stagnation pressure for convenience) as

$$
u^{s}=C U^{s}, \quad w^{s}=C W^{s} \text { and } q^{s}=\left(\left(u^{s}\right)^{2}+\left(w^{s}\right)^{2}\right) / 2+C^{2} P^{s},
$$

and take into account the solution (4.3) of (3.11), (3.18) and (3.20) to rewrite (3.28)(3.32) as

$$
\begin{aligned}
& U_{r}^{s}+r^{-1} U^{s}+W_{z}^{s}=0, \\
& U^{s} U_{r}^{s}+W^{s} U_{z}^{s}=-P_{r}^{s}+U_{r r}^{s}+r^{-1} U_{r}^{s}-r^{-2} U^{s}+U_{z z}^{s}, \\
& U^{s} W_{r}^{s}+W^{s} W_{z}^{s}=-P_{z}^{s}+W_{r r}^{s}+r^{-1} W_{r}^{s}+W_{z z}^{s}, \\
& U^{s}=W^{s}=0 \text { at } z= \pm A, \quad U^{s}=W_{r}^{s}=P_{r}^{s}=0 \quad \text { at } r=0, \\
& U^{s}=W_{r}^{s}+K\left(\mathrm{e}^{8 \omega z / 3}-\delta \mathrm{e}^{-8 \omega z / 3}\right)=0 \quad \text { at } r=1,
\end{aligned}
$$


where $\omega=\Omega C$ (see (3.12)) and

$$
K=8 \varepsilon^{2} \Omega k^{2}\left|A_{0}\right|^{2} / C>0, \quad \text { and } \delta=\left|B_{0}\right|^{2} /\left|A_{0}\right|^{2}>0,
$$

with $A_{0}$ and $B_{0}$ given by (4.4). Notice that for a fixed value of the capillary Reynolds number $C^{-1}$, the positive parameter $\omega$ may be arbitrarily chosen by selecting the vibrating frequency $\Omega$ and, according to the remark at the end of $\$ 4$, the positive parameters $K$ and $\delta$ may be also arbitrarily chosen by selecting (in a non-unique way) the vibrating amplitudes of the disks and the phase shift. Also, we must take into account the limitations derived in $\$ 3.3$, which may be written as $C \ll$ $\omega \ll C^{-2}$ and $K \ll \max \left\{\Omega^{2}, \Omega^{-1 / 2} C^{-3 / 2\}}\right.$ (the second limitation may be relaxed to $K \ll \max \left\{\Omega^{2}, C^{-1} \Omega^{1 / 3}\right\}$ when the steady streaming flow is symmetric). If we assume that $10^{2} \leqslant C^{-1} \lesssim 10^{4}$ then $\omega$ varies over a wide range and $K$ may be safely taken as large as $10^{3}$. The asymmetry parameter $\delta$ will vary between 0 and 1 (for the problem is invariant under the transformation $\delta \rightarrow 1 / \delta, K \rightarrow K \delta, z \rightarrow-z$ and $W \rightarrow-W$ ).

The problem (5.1)-(5.5) is numerically solved below as follows. The pressurevelocity formulation of the flow field is considered and the discretization is made according to the finite volume method of Patankar (1980). The pressure-correction method (Connel \& Stow 1986) is used to solve the discretized equations obtained from the governing equations. In particular, the SIMPLEC approximation of Van Doormaal \& Raithby (1984) is used. A central difference scheme is used to discretize the governing equations (Minkowycz et al., 1988). The computational domain is divided into small control volumes, whose size follows a logarithmic distribution that concentrates the control volumes near the disks, their mid-plane, the centre of the liquid bridge and the interface (i.e. in the steady viscous boundary layers when the Reynolds number associated with the flow is large). The centres of these control volumes form the grid used for the pressure, and the staggered grid system of Harlow \& Welch (1965) is considered for the components of the velocity vector. In the iterative procedure, convergence is declared when the three variables, $U^{s}, W^{s}$ and $P^{s}$ satisfy simultaneously the relative error criterion

$$
\sum\left|g_{i j}-g_{i j}^{0}\right| / \sum\left|g_{i j}\right|<5 \times 10^{-5},
$$

where the summation is over all control volumes, and $g_{i j}^{0}$ stands for the value at the previous step. Also, in the nomenclature of Van Doormaal \& Raithby (1984), the value $E=8.0$ is used for the relaxation parameter, and the number of control volumes in $[-A, A] \times[0,1]$ is $200 \times 100$.

The solution of (5.1)-(5.5), depends only on the positive vibrational parameters $\omega$, $K$ and $\delta$ that control respectively the steepness, the strength and the asymmetry of the forcing tangential stress at the interface. Several parameter ranges are now considered. For brevity, only the most representative numerical results will be explicitly given.

(a) If $\omega \ll 1$ (i.e. if $\Omega \ll C^{-1}$, see (3.12)) then the steady forcing tangential stress at the interface is approximately constant (and equal to $-K(1-\delta)$, see (5.5)) and the steady streaming flow exhibits a unique toroidal eddy whose rotation sense may be controlled by means of the asymmetry parameter $\delta$ (which in turn may be controlled by the phase shift between the vibration of both disks, see the remark at the end of $\$ 4$ and (5.6)). For illustration, the axial velocity at the interface and the streamlines are given with solid lines in figure 5 for the particular case $A=1, \omega=0$ and $K(1-\delta)=10^{3}$. For smaller values of $K(1-\delta)$ the effect of convective terms decreases, the eddy moves to the centre of the liquid bridge and the axial velocity at the interface becomes more symmetric. That value of $K(1-\delta)$ may be obtained, for 

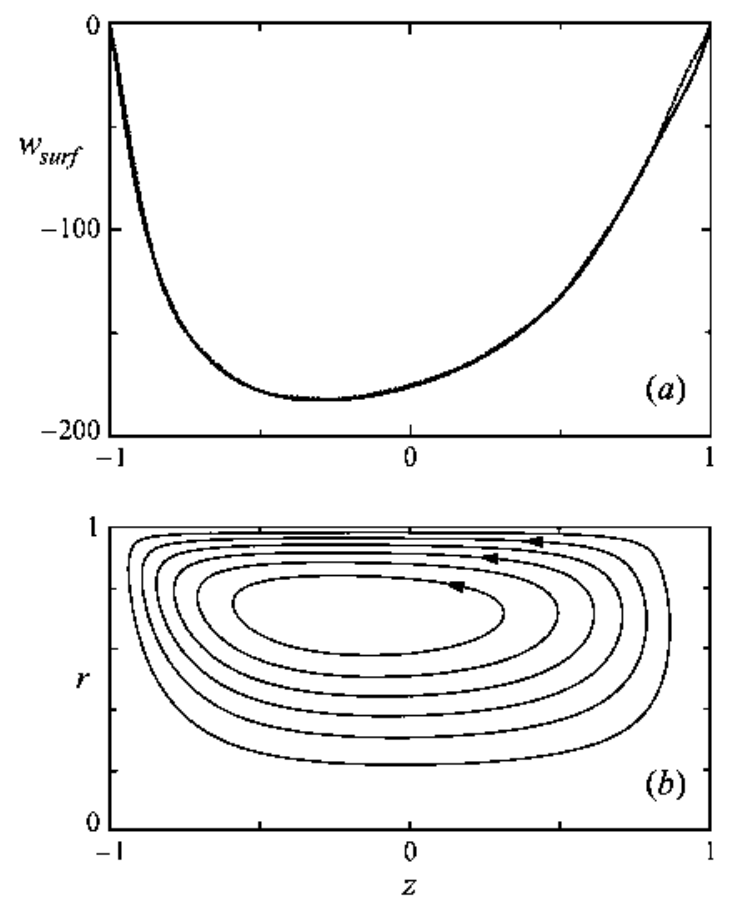

FiguRE 5. Comparison of high-frequency (solid lines) and low-frequency (dotted lines) models: (a) free-surface velocily, (b) streamlines.

example, if $\Omega=104$ (that is between two consecutive inviscid eigenfrequencies, see table 1 ), $\beta_{+}=\mathrm{i} \beta_{-}$and

$$
\varepsilon^{2}\left|\beta_{+}\right|^{2} / C=\left[10^{3} / 8 \Omega k^{2}\left(\gamma_{1}^{2}-\gamma_{2}^{2}\right)\right] \sin (2 v+A / 3) \simeq 6.19 \times 10^{-4},
$$

as obtained when taking into account (4.11), (5.6) and that $v \simeq 21.0679, \gamma_{1} \simeq 1.9912$ and $\gamma_{2} \simeq 0.01175$, according to (A 21). Notice that the required vibration amplitude of the disks is extremely small, $\varepsilon\left|\beta_{+}\right| \simeq 2.49 \times 10^{-2} C^{1 / 2}=7.87 \times 10^{-4}$ if $C=0.001$. With these particular values of the vibrational parameters we may calculate the steady streaming flow by means of the asymptotic model derived by Nicolás et al. (1997) that applies whenever $\Omega \ll C^{-1}$ (without the assumption $\Omega \gg 1$ ). The resulting axial velocity at the interface and the streamlines are plotted with dashed lines in figure 5. Notice that both approximations yield approximately the same flow, in spite of the fact that the forcing tangential stress at the interface is significantly different (the tangential stress in Nicolás et al. 1997 exhibits a fast oscillation around a constant value while here it is approximately constant); this was to be expected because the oscillation only yields a stationary wave that does not penetrate the bulk and exhibits an axial velocity that is small compared to the axial velocity in the bulk, as seen from the analysis in $\$ 3.2$ above. These asymmetric flows are the appropriate ones to counterbalance thermocapillary flows in half-zone liquid bridges (i.e. in liquid bridges with the disks held at two different temperatures and the interface thermally insulated), as was seen by Nicolás et al. (1997).

(b) If $\omega \neq 0$ and $\delta=1$ then the flow pattern is symmetric and consists of a pair of counter-rotating toroidal eddies that push the liquid along the interface from the disks towards the centre of the liquid bridge. As either $\omega$ or $K$ increases, the strength of the 

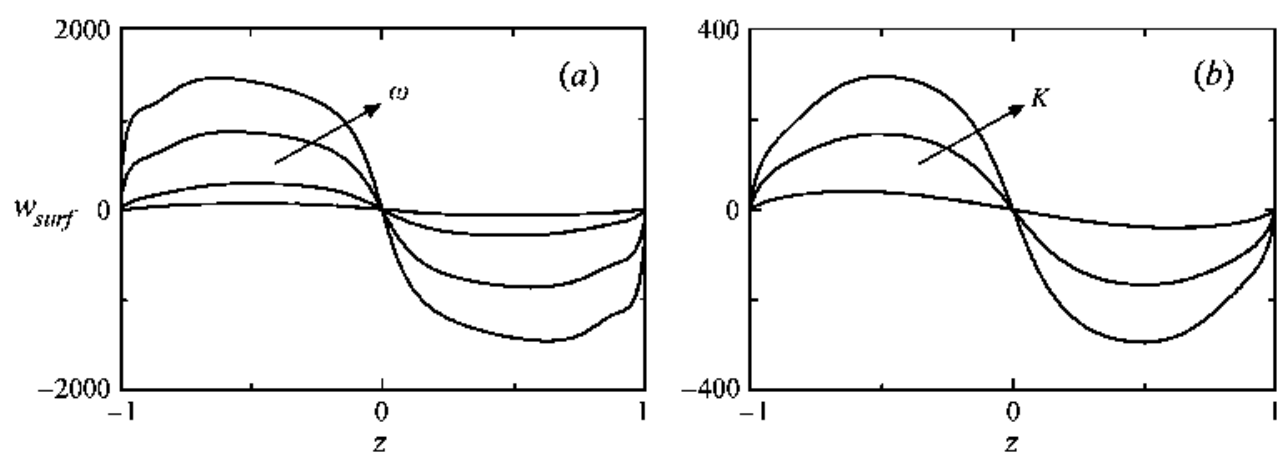

FIGUkE 6. Free-surface velocity for $A=1, \delta=1$. (a) $K=1000$ and $8 \omega / 3=0.5,2,4$ and 5 .

(b) $80 \% / 3=2$ and $K=100,500$ and 1000 .

steady streaming flow increases, as illustrated in figure 6 where the axial velocity at the interface is plotted (i) for $K=10^{3}$ and several values of $\omega$, and (ii) for $8 \omega / 3=2$ and several values of $K$; the associated streamlines are not plotted because they are qualitatively similar to each other (and to those in figure $7 b$ below). Of course, as the steepness parameter $\omega$ increases, the forcing tangential stress at the interface becomes more and more localized near the disks and the two counter-rotating eddies should move towards the disks. But in order to appreciate this effect the parameter $K$ must be varied in such a way that the net forcing tangential stress at the interface, $\int_{-A}^{A}\left|w_{r}(1, z)\right| \mathrm{d} z$, remains constant (for, if instead $K$ remains constant then convection increases as $\omega$ increases, the eddies are pushed away from the disks by convective effects and this counterbalances the localization effect). This is illustrated in figure 7 , where $\int_{-A}^{A}\left|w_{r}(1, z)\right| \mathrm{d} z=100$ for all values of $\Omega$.

(c) If $\omega \neq 0$ then the flow pattern is as asymmetric as possible when $\delta=0$. In this case the flow pattern exhibits a unique toroidal eddy that pushes the liquid along the interface from $z=A$ to $z=-A$. As in case $(b)$, the strength of the steady streaming flow increases as either $\omega$ or $K$ increases, as illustrated in figure 8; the associated streamlines are not plotted because they are again similar to each other and to those in figure $9(b)$ below. If the net forcing tangential stress at the interface, $\int_{-A}^{A}\left|w_{r}(1, z)\right| \mathrm{d} z$, is kept constant and the steepness parameter $\omega$ is increased then the eddy moves towards the corner $r=1, z=A$, as happened in case $(b)$ above, but for brevity we do not plot streamlines showing this effect. These asymmetric flows are not the appropriate ones to counterbalance asymmetric thermocapillary convection in half-zone liquid bridges because now the forcing tangential stress (due to vibration) at the free surface is far from being constant (unless $\omega \ll 1$, but this limit leads us to case (a) above).

(d) If $\omega \neq 0$ and $0<\delta<1$ then the flow pattern is between those described in the limits $(b)$ and (c) above, and consists of a pair of unequal counter-rotating toroidal eddies that push the liquid along the interface away from the disks, with the smaller eddy increasing as $\delta$ increases. For illustration, the axial velocity at the interface and the streamlines are plotted in figure 9 for $A=1,8 \omega / 3=2, K=10^{3}$ and five representative values of $\delta$. As $\omega$ and/or $K$ vary the conclusions in case (b) above stand.

In order to illustrate the quantitative dependence of the streaming flow on the forcing amplitude, the maximum value of the axial velocity at $r=1$ has been plotted vs. $K$ (which in turn is proportional to the square of the vibrating amplitude, see (4.4) 

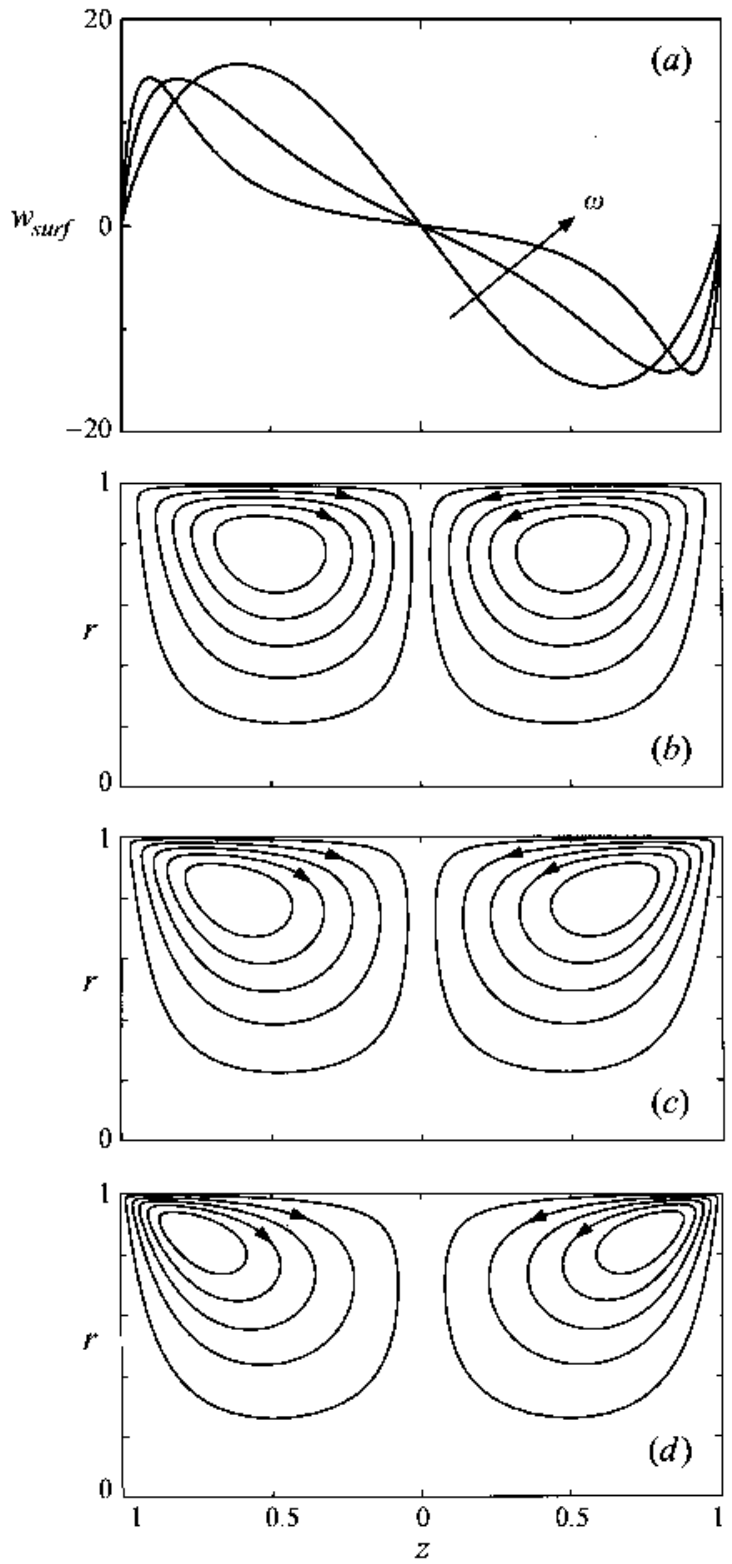

FIGURE 7. (a) Free-surface velocity for the values of the parameters in (b) (d). (b) (d) Streamlines for $A=1, \delta=1$ and $(\omega, K)=(0.5,195.9),(5,3.415)$ and $(10,0.04540)$ respectively.

and (5.6)) in figure 10, in two particular cases. The plot in figure $10(a)$ corresponds to the case when only one disk is vibrated (for instance, that near $z=A$ and then $\beta_{+} \neq 0, \beta_{-}=0$ ), with $A=1$ and $\omega=\Omega C=3 / 4$. In addition we have taken $C=10^{-4}$. Then $\Omega=\frac{3}{4} \times 10^{4}$ and $K$ and $\delta$ are calculated from (A 21), (4.10) and (5.6) to be

$$
K \simeq 1.4765 \times 10^{14} \varepsilon^{2}\left|\beta_{+}\right|^{2}, \quad \delta \simeq 0.0180 .
$$

Notice that even for $K=4 \times 10^{4}$ (which corresponds to a quite large value of the effective Reynolds number associated with the streaming flow) the vibrating amplitude 

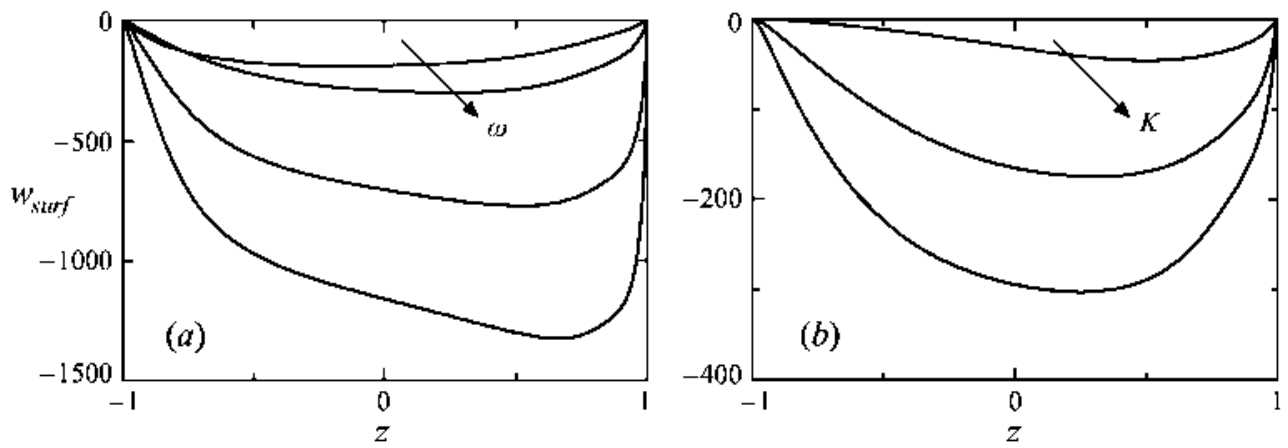

FIGURE 8. Frec-surface velocity for $A=1, \delta=0$. (a) $K=1000$ and $8 \omega / 3=0.5,2,4$ and 5 . (b) $8 \omega / 3=2$ and $K=100,500$ and 1000 .
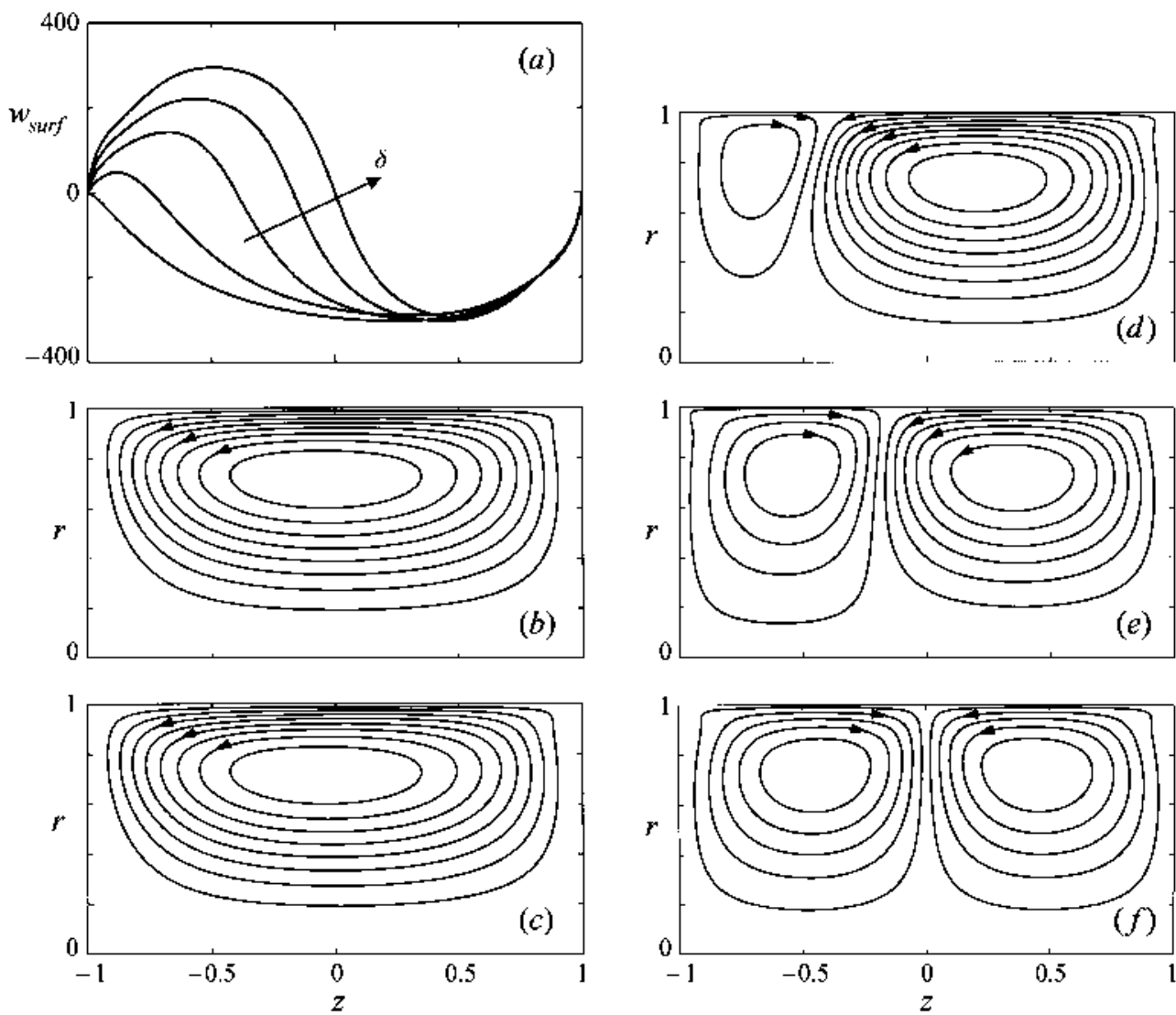

FIGURF 9. (a) Free-surface velocity for the values of the parameters in $(b)-(f) .(b)$ - $(f)$ Streamlines for $A=1, K=1000,8 \omega / 3=2$ and $\delta=0,0.25,0.5,0.75$ and 1 respectively.

is quite small, and the limitations in $\$ 3.3$ still hold. This plot shows that if $K \leqslant 10^{2}$ then the velocity is proportional to $K$, while for $K \gtrless 10^{3}$ the velocity is proportional to $K^{2 / 3}$, as anticipated in $\$ 3.3$. The plot in figure $10(b)$ corresponds to the case when both disks are vibrated either in phase or in counterphase (then $\beta_{+}= \pm \beta_{-}$), with 

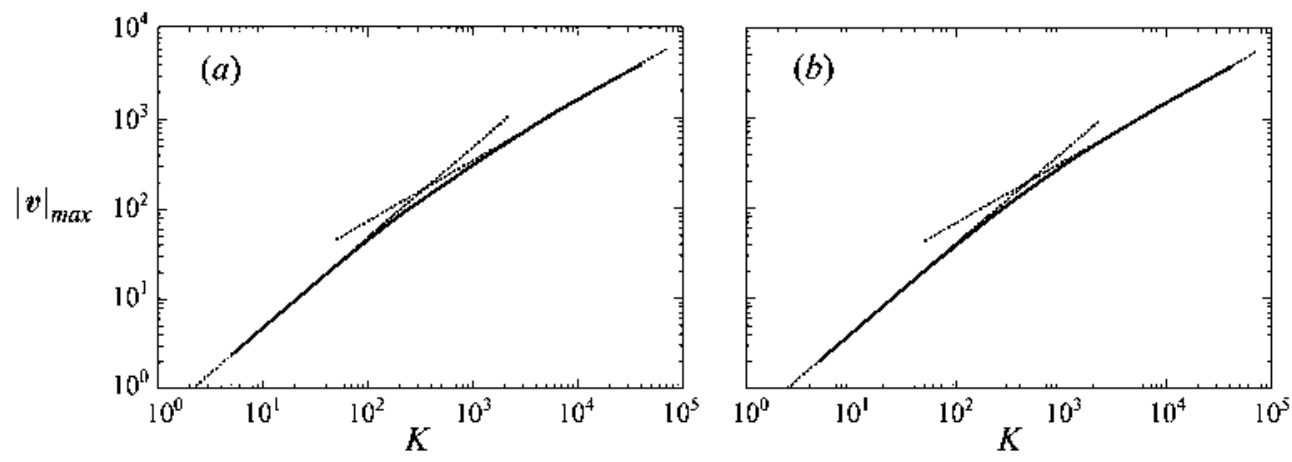

FIGUkE 10. Maximum velocity modulus as a function of $K$, for $A=1$ and $8 \omega / 3=2$. (a) Vibration of onc disk $(z=+A): \delta=0.0180$. (b) Vibration of both disks in phase or counterphase: $\delta=1$. Dotted lines correspond to asymptotes of slope 1 and $2 / 3$.

$A=1$ and $\omega=3 / 4$. If, as above, we take $C=10^{-4}$ and $\beta_{+}=\beta_{-}$then

$$
K \simeq 1.6608 \times 10^{14} \varepsilon^{2}\left|\beta_{+}\right|^{2}, \quad \delta=1,
$$

and exactly the same comments made above (in connection with figure 10a) apply again.

\section{Concluding remarks}

We have considered the linear oscillating flow and the associated nonlinear steady streaming flow resulting from high-frequency axial vibrations of a nearly inviscid axisymmetric capillary bridge; we considered the distinguished limit $\Omega \sim C^{-1} \gg 1$, where $\Omega$ is the non-dimensional forcing frequency based on the capillary time, and $C^{-1}$ is the capillary Reynolds number, but our analysis applies whenever $1 \ll \Omega \ll C^{-3}$ (two additional, essentially different distinguished limits apply as $\Omega \sim 1$ and as $\Omega \sim C^{-3}$, as explained in $\$ 1$ ).

The leading oscillatory flow was obtained in $\$ 3.1$, where up to six different regions were considered in the liquid bridge. In particular, this flow was seen to exhibit a $O$ (1)-wavelength in the bulk and a wavelength $k^{-1}=\Omega^{-3 / 2} \ll 1$ in a thin (with a thickness of the order of $k^{-1}$ ) nearly inviscid sublayer attached to the free surface, where the oscillating flow consists of two counterpropagating wavetrains that travel with a phase velocity $\Omega / k=k^{1 / 2} \gg 1$ and do not penetrate the bulk. The steady complex amplitudes $A$ and $B$ of these wavetrains were seen to obey a pair of amplitude equations that are balances between a convective term accounting for the group velocity (which equals $3 k^{1 / 2} / 2 \gg 1$ ), viscous damping (which yields a spatial decay of the waves as they travel along the free surface) and a first correction to the wavenumber (which produces a spatial variation of the local phase of the wavetrains). We neglected nonlinearity (which includes a coupling effect of the streaming flow on the vibrating flow), dispersion and other higher-order effects that were discussed in \$3.3. The wavetrains are reflected and forced in two nearly-inviscid corner regions near the edges of the disks whose analysis, in $\$ 3.1$, shows that the waves are purely reflected (i.e. no part of the incoming wave is absorbed) to a first approximation, and that the outgoing wave is forced by two mechanisms, namely, a direct forcing from the nearby vibrating disk and a forcing from the bulk; the first mechanism is weakly dominant (by a factor of the order of $\log k \gg 1$ ) and local, while the 
second mechanism is non-local (i.e. forcing near one disk depends on the vibration parameters of the other disk). The amplitude equations and boundary conditions were discussed in $\$ 4$ where it was seen that (i) our asymptotic approximations to the damping rate, the reflection coefficient and the forcing mechanisms give quite good results for not-large values of $\Omega$ and (ii) the quantitative shape of $A$ and $B$ is quite sensitive to the vibration parameters (interference properties of the incoming and outgoing waves near the disks playing a crutial role). Thus, the steady tangential stress at the edge of the nearly inviscid sublayer, $8 \varepsilon^{3} \Omega k^{2}\left(|A|^{2}-|B|^{2}\right)$ (see (3.35)), which drives the streaming flow in the bulk, is also quite sensitive to the vibration parameters. Thus, our careful analysis of the leading oscillating flow in $\$ 3.1$ and $\$ 4$ (which could be seen as secondary in this paper, albeit of its intrinsic interest) is essential in order to get a quantitatively good approximation of the streaming flow in terms of the parameters associated with the vibration of the disks (i.e. amplitudes, frequencies and phase shift), which are the actual input of the physical problem.

The steady streaming flow was considered in $\$ 3.2$ (where the steady tangential stress at the internal edge of the nearly inviscid interface sublayer was obtained), $\$ 3.3$ (where the asymptotic model was derived) and $\$ 5$ (where the streaming flow structure was numerically calculated and discussed). In particular, we have seen that the flow is only driven by the tangential stress resulting from the viscous oscillatory boundary layer near the interface; the forcing tangential velocity at the edge of the Stokes boundary layers near the disks has been seen to be a higher-order effect in this limit. The model (3.11), (3.18), (3.20) and (3.28)-(3.32) was obtained in terms of the time-averaged velocity, $\left(t^{s}, w^{s}\right)$, that was seen (at the end of $\left.\$ 3.3\right)$ to coincide to a first approximation with the drift velocity, associated with the time-averaged trajectories of material elements. We have neglected in the momentum equations forcing averaged terms resulting from the time average of products of first- and third-order oscillatory terms in convective terms; this assumption was justified in \$3.3. where we also considered the assumptions associated with our assuming that the thickness of the oscillatory, interface boundary layer is small compared to the thickness of the nearly inviscid sublayer, and that the latter is small compared to the thickness of the possible viscous steady boundary layers of the streaming flow (when its effective Reynolds number is large).

Our discussion in $\$ 5$ shows that both the strength and qualitative shape of the streaming flow can be controlled quite effectively through the appropriate selection of the vibration parameters. In particular, a large variety of symmetric and nonsymmetric flows, with varying intensity in their localization near the disks (or the centre of the free surface) are obtained. This is a quite important difference of this limit with the remaining two asymptotic limits considered in $\$ 1$, which by no means exhibit that wealth of behaviour.

Finally, let us make a brief comment of the ability of the streaming flows obtained in $\$ 5$ to annihilate thermocapillary flows. Qualitatively antisymmetric thermocapillary flows (resulting from, e.g. fixing the temperatures of the disks, with a thermally insulated free surface) may be quite effectively controlled in the limit $\omega=\Omega C \ll 1$, as explained in $\$ 5$, case $(a)$; this is not surprising because of the results by Nicolás $e t a l$. (1997). The interest of this result is somewhat limited because these thermocapillary flows apply only in experiments. The flows appearing in the melt when using the floatzone technique instead are essentially symmetric because they result from a more or less symmetric heating of the interface, and cannot be annihilated in the other two limits considered in $\$ 1$. On the contrary, they can be annihilated with the streaming flows studied in this paper because, if (as usually happens in practice) surface tension 
decreases with temperature, then thermocapillary stress pushes the liquid along the interface from the centre towards the disks and this is precisely opposite to what tangential stress resulting from vibration does (see figure $7 \mathrm{~b}$ ).

This research was supported by DGICYT and by the EEC Program on Human Capital and Mobility, under Grants PB-93-0046 and CHRX-CT-93-0413.

\section{Appendix A. Some algebraic expressions from $\$ 3$}

The unique solution of the linear problem (3.2)-(3.3) can be decomposed into its odd and even parts, as

$$
\left.\begin{array}{rl}
U & =\left[\left(\beta_{+}+\beta_{-}\right) U^{o}+\left(\beta_{+}-\beta_{-}\right) U^{e}\right] / 2, \\
W & =\left[\left(\beta_{+}+\beta_{-}\right) W^{o}+\left(\beta_{+}-\beta_{-}\right) W^{e}\right] / 2, \\
Q & =\left[\left(\beta_{+}+\beta_{-}\right) Q^{o}+\left(\beta_{+}-\beta_{-}\right) Q^{e}\right] / 2,
\end{array}\right\}
$$

that are readily calculated in semi-analytical form as

$$
\begin{aligned}
U^{o} & =Q_{r}^{o}, \quad U^{e}=Q_{r}^{e}, \quad W^{o}=Q_{z}^{o}, \quad W^{e}=Q_{z}^{e}, \\
Q^{o} & =z+\frac{2}{A} \sum_{m \text { odd }} \frac{I_{0}\left(l_{m} r\right)}{l_{m}^{2} I_{0}\left(l_{m}\right)} \cos \left(l_{m}(z+A)\right), \\
Q^{e} & =\frac{3-2 A^{2}-3 r^{2}+6 z^{2}}{12 A}-\frac{2}{A} \sum_{m \text { even }} \frac{I_{0}\left(l_{m} r\right)}{l_{m}^{2} I_{0}\left(l_{m}\right)} \cos \left(l_{m}(z+A)\right),
\end{aligned}
$$

where $I_{0}$ and $I_{1}$ are the first two modified Bessel functions and $l_{m}=m \pi / 2 A$, for $m=1,2, \ldots$.

The second-order problem giving the terms $u_{2}, w_{2}, q_{2}$ and $f_{2}$ appearing in the expansions (3.6) and (3.7) (for the oscillatory solution in region (b)) are given by

$$
\begin{aligned}
& -u_{2 \xi}+w_{2 \eta}+u_{1}+w_{1 z}=u_{2}+q_{2 \xi}=w_{2}-q_{2 \eta}-q_{1 z}=0, \\
& u_{2}=w_{2}=q_{2}=0 \text { at } \xi=\infty, \\
& u_{2}-f_{2}-2 \mathrm{i} \omega f_{1 \eta \eta}=q_{2}+f_{2 \eta \eta}+2 f_{1 \eta z}+2 \mathrm{i} \omega u_{1 \xi}=0 \text { at } \xi=0,
\end{aligned}
$$

where (see (2.11))

$$
\omega=\Omega C \sim 1,
$$

and in (A 6) and (A 7) we are using matching conditions with regions (a) and (d) (see (B 3) and (B 5) in Appendix B).

Notice that there are two terms proportional to $\omega$ (which will yield a counterpart accounting for viscous dissipation in (3.11) below) in the boundary conditions (A 7). The first one, $-2 \mathrm{i} \omega f_{1 \eta n}$, comes (through matching conditions) from the effect of the oscillatory viscous interface boundary layer, and the second one, $2 \mathrm{i} \omega t_{1 \xi}$, directly comes from the viscous normal stress at the free surface (see $(2.8)$ ); notice that if the first term is ignored (as frequently done in the literature, when a careful analysis of the interface boundary layer is avoided) then the damping rate is underestimated by a factor of $1 / 2$. Now we only need to require the solution of (A 5)-(A 7) to be bounded, as either $\xi \rightarrow \infty$ or $|\eta| \rightarrow \infty$, to obtain the following solvability conditions:

$$
3 A_{z}-(4 \omega+\mathrm{i} / 2) A=3 B_{z}+(4 \omega+\mathrm{i} / 2) B=0 .
$$

When taking into account (A 1)-(A 4), (3.5), (3.7) and (3.10), the following asymptotic 
behaviour of $f$ is readily obtained:

$$
\begin{aligned}
& f-1= \pm \varepsilon \mathrm{e}^{\mathrm{i} \Omega t} {\left[\left(\frac{2}{\pi} \log (A \mp z)+\delta_{1}\right) \beta_{ \pm}+\delta_{2} \beta_{\mp}\right] } \\
&+\varepsilon A( \pm A) \mathrm{e}^{\mathrm{i} \Omega t \pm \mathrm{i} k A \mp \mathrm{i} k(A \mp z)}+\varepsilon B( \pm A) \mathrm{e}^{\mathrm{i} \Omega t \mp \mathrm{i} k A \pm \mathrm{i} k(A \mp z)}+\text { c.c. }+ \text { HOT, }
\end{aligned}
$$

as $z \rightarrow \pm A$, where HOT stands for higher-order terms and the real constants $\dot{\delta}_{1}$ and $\delta_{2}$ are

$$
\left.\begin{array}{l}
\delta_{1}=\frac{2}{\pi} \log \frac{\pi}{2 A}-\frac{1}{4 A}+\frac{1}{A} \sum_{m=1}^{\infty} \frac{I_{0}\left(l_{m}\right)-I_{1}\left(l_{m}\right)}{l_{m} I_{0}\left(l_{m}\right)}, \\
\delta_{2}=-\frac{2}{\pi} \log 2+\frac{1}{4 \Lambda}-\frac{1}{A} \sum_{m=1}^{\infty}(-1)^{m} \frac{I_{0}\left(l_{m}\right)-I_{1}\left(l_{m}\right)}{l_{m} I_{0}\left(l_{m}\right)} .
\end{array}\right\}
$$

The linear problem (3.15) and (A 12) (giving the leading-order part of the oscillatory solution in region (c) near $r=1, z=A$ ) is solved via sine- and cosine-Fourier transforms as

$$
\begin{aligned}
& \check{q}_{1}=a_{1}\left(\xi+\mathrm{e}^{-\xi} \cos \tilde{\eta}\right) \\
& +a_{2}\left[\frac{2}{\pi} \int_{0}^{\infty}\left(\frac{\mathrm{e}^{-k \tilde{\eta}} \sin k \xi}{k^{2}+1}+\frac{k^{2} \mathrm{e}^{-k \xi} \cos k \tilde{\eta}}{(k+1)\left(k^{3}-1\right)}\right) \mathrm{d} k+\mathrm{e}^{-\xi}\left(\sin \tilde{\eta}+\frac{2 \sqrt{3}}{9} \cos \tilde{\eta}\right)\right] \\
& +\beta_{+}\left[\frac{2}{\pi} \int_{0}^{\infty} \frac{\mathrm{e}^{-k \xi} \cos k \tilde{\eta}+\xi k \cos k+k^{3}-1}{k^{2}\left(k^{3}-1\right)} \mathrm{d} k-b_{1} \mathrm{e}^{-\xi} \cos \tilde{\eta}-\tilde{\eta}\right], \\
& \tilde{f}_{1}=a_{1}(\cos \tilde{\eta}-1)+a_{2}\left[\frac{2}{\pi} \int_{0}^{\infty} \frac{\cos k \tilde{\eta} \mathrm{d} k}{(k+1)\left(k^{3}-1\right)}+\sin \tilde{\eta}+\frac{2 \sqrt{3}}{9} \cos \tilde{\eta}\right] \\
& +\beta_{+}\left(\frac{2}{\pi} \int_{0}^{\infty} \frac{\cos k \tilde{\eta}-\cos k}{k\left(k^{3}-1\right)} \mathrm{d} k-b_{1} \cos \tilde{\eta}\right),
\end{aligned}
$$

where the principal value must be taken in divergent integrals, $a_{1}$ and $a_{2}$ are arbitrary complex constants and

$$
b_{1}=\frac{2}{\pi} \int_{0}^{x_{1}} \frac{(1-\cos k) \mathrm{d} k}{k\left(k^{3}-1\right)} \simeq 0.043476 .
$$

For matching purposes we only need the asymptotic behaviour of $\tilde{f}_{1}$ as $\tilde{\eta} \rightarrow \infty$, which is found to be given by

$$
\begin{aligned}
& \hat{f}_{1}=\left(2 \beta_{+} / \pi\right) \log \tilde{\eta}-\left(a_{1}-\beta_{+} b_{2}\right)+\left[a_{1}+\right.\left.(2 \sqrt{3} / 9) a_{2}-\beta_{+} b_{1}\right] \cos \tilde{\eta} \\
&+\left[2\left(a_{2}-\beta_{+}\right) / 3\right] \sin \tilde{\eta}+o(1),
\end{aligned}
$$

where

$$
b_{2}=b_{1}+2 \gamma / \pi \simeq 0.41094 ;
$$

$\gamma \simeq 0.577215$ is the Euler constant and we have taken into account that

$$
\int_{0}^{\infty} \frac{(k-1) \mathrm{d} k}{(k+1)\left(k^{2}+k+1\right)}=0 .
$$


Now we only need to take into account (A 10), (3.13), (3.14) and (A 15), and apply a matching condition between both expressions for $f$ to obtain

$$
\begin{gathered}
a_{1}=\left[(2 / \pi) \log k+b_{2}-\delta_{1}\right] \beta_{+}-\delta_{2} \beta_{-}, \\
A(A) \mathrm{e}^{\mathrm{i} k A}+B(A) \mathrm{e}^{-\mathrm{i} k A}=a_{1}+(2 \sqrt{3} / 9) a_{2}-\beta_{+} b_{1}, \\
A(A) \mathrm{e}^{\mathrm{i} k A}-B(A) \mathrm{e}^{-\mathrm{i} k A}=2 \mathrm{i}\left(a_{2}-\beta_{+}\right) / 3,
\end{gathered}
$$

where the complex constant $a_{2}$ remains arbitrary. If $a_{2}$ is eliminated in (A 18) and (A 19), and $a_{1}$ is substituted from (A 17), then the following equation results:

$$
A(A)=\mathrm{e}^{-2 \mathrm{iv}} B(A)-\mathrm{ie}^{-\mathrm{i} v}\left(\gamma_{1} \beta_{+}-\gamma_{2} \beta_{-}\right)
$$

where

$$
v=k A-\pi / 3, \quad \gamma_{1}=\sqrt{3}\left[(2 / \pi) \log k-b_{1}+b_{2}-\delta_{1}\right] / 2+1 / 3, \quad \gamma_{2}=\sqrt{3} \delta_{2} / 2,
$$

with $\delta_{1}, \delta_{2}, b_{1}$ and $b_{2}$ as given by (A 11), (A 14) and (A 16).

Appendix B. The viscous boundary layers (d), (e) and (f)

The size of these regions is of the order of $(\Omega / C)^{1 / 2}$, such that inertia and viscous terms are comparable in momentum conservation equations. As pointed out at the begining of $\$ 3$ we omit the (fairly involved) analysis of these regions; it is briefly summarized here.

In the interface boundary layer (d) we use the slow variable $z$ and the fast stretched variables

$$
\zeta=(\Omega / C)^{1 / 2}(f-r) \text { and } \eta=k z \text {, }
$$

that define a moving coordinate system attached to the interface. In addition, we use the associated dependent variables $u^{*}, w^{*}, p^{*}$ and $f^{*}$, defined as

$$
\left.\begin{array}{c}
u=f_{t}+\left(k f_{\eta}+f_{z}\right) w+\varepsilon k(\Omega C)^{1 / 2} u^{*}, \quad w=\varepsilon \Omega w^{*} \\
q=1+\left(u^{2}+w^{2}\right) / 2+\varepsilon k^{2} p^{*}, \quad f=1+\varepsilon f^{*},
\end{array}\right\}
$$

where $f^{*}=\mathrm{e}^{\mathrm{i} \Omega t}\left[U(1, z)+f_{1}+k^{-1} f_{2}+\right.$ HOT $]+$ c.c. + NRT (see (3.7)). When these new variables and the appropriate expansions for both the oscillatory and the steady parts of $u^{*}, w^{*}$ and $q^{*}$ are inserted into $(2.1)-(2.3)$ and $(2.5)-(2.8)$, the resulting problems are solved and the limit $\zeta \rightarrow \infty$ is considered, the following asymptotic behaviour for the solution in this region is found:

$$
\begin{aligned}
& u=\varepsilon \Omega \mathrm{ie}^{\mathrm{i} \Omega t}\left[U+f_{1}-k(C / \Omega)^{1 / 2} \zeta f_{l \eta \eta m}+k^{-1}\left(f_{2}+2 \mathrm{i} \omega f_{\perp \eta \eta}\right)\right]+\text { c.c. } \\
& +\varepsilon^{2} \Omega k\left(i f_{1 \eta} \bar{f}_{1 m m \eta n}+\text { c.c. }\right)+\text { NROT }+ \text { HOT, } \\
& w_{\zeta}=-\varepsilon k(C / \Omega)^{1 / 2}\left[\mathrm{i} \Omega \mathrm{e}^{\mathrm{i} \Omega t} f_{1 \eta}+\text { c.c. }+\varepsilon k \Omega\left(6 \mathrm{i} f_{1 \eta} \bar{f}_{1 \eta \eta \eta \eta}+\mathrm{i} f_{1 \eta \eta} \bar{f}_{1 \eta \eta \eta \eta}+\text { c.c. }\right)\right] \\
& \text { +NROT + HOT, } \\
& q-\mathbf{1}=-\varepsilon k^{2}\left[\mathrm{e}^{\mathrm{i} \Omega t}\left(-f_{1 \eta \eta}+k(\Omega / C)^{1 / 2} \zeta\left(U(1, z)+f_{1}\right)+k^{-1}\left(f_{2 \eta \eta}+2 f_{1 \eta z}-2 \mathrm{i} \omega f_{1 \eta \eta \eta \eta}\right)\right)\right. \\
& + \text { c.c. }]+\mathrm{HOT}+\mathrm{NRT} \text {, }
\end{aligned}
$$

as $\zeta \rightarrow \infty$, where NROT, NRT and HOT stand for non-resonant oscillatory terms, 
(depending on $t$ as $\exp (\mathrm{i} m \Omega t$ ), with $m \neq \pm 1,0$ ), non-resonant terms (depending on $t$ as $\exp \mathrm{i} m \Omega t$, with $m \neq \pm 1$ ) and higher-order terms than those displayed respectively.

A similar but much simpler analysis of the Stokes boundary layers (e) shows that the solution in these regions satisfies

$$
\begin{gathered}
u=O\left(\varepsilon^{2} \Omega\right)+\mathrm{NST}, \\
w=\varepsilon \mathrm{e}^{\mathrm{i} \Omega t}\left(\mathrm{i} \Omega \beta_{ \pm}+\mathrm{HOT}\right)+\text { c.c. }+O\left(\varepsilon^{2} \Omega\right)+\mathrm{NROT},
\end{gathered}
$$

where NROT and HOT are as above, and NST stands for non-steady terms (depending on $t$ as $\exp (\mathrm{i} m \Omega t)$, with $m \neq 0$ ).

The oscillatory viscous regions (f) provide higher-order terms, that need not be considered.

\section{REFERENCES}

Anilkumar. A. V., Grugel. R. N.. Shen, X. F., Lee, C. P. \& Wang, T. G. 1993 Control of thermocapillary convection in a liquid bridge by vibration. Appl. Phys. 73, 4165-4170.

BaTchelor, G. K. 1967 An Introduction to Fluid Dynamics. Cambridge University Press.

Biringen, S. \& Peltier, L. J. 1990 Computational study of 3-D Bénard convection with gravitational modulation. Phys. Fluids A 2, 279-283.

ConNell, S. D. \& STow, P. 1986 The pressure correction method. Computers Flitis 14, 1-10.

Craik, A. D. D. 1982 The drift velocity of water waves. J. Fluid Mech. 116, 187-205.

CRöll, A., Dold, P. \& Benz, K. W. 1994 Segregation of Si floating-zone crystals grown under microgravity and in a magnetic field. $J$. Cryst. Growth 137, 95-101.

Davey, A. \& StewarTson, S. 1974 On three-dimensional packets of surface waves. Proc. R. Soc. Lond. A 338, 101-110.

DAviAud, F. \& VINCE. J. M. 1993 Travelling waves in a flujd layer subjected to a horizontal temperature gradient. Phys. Rev. E 48, 4432-4436.

Dressler, R. F. \& Sivakumaran, N. S. 1988 Non-contaminating method to reduce Marangoni convection in microgravity float zones. J. Cryst. Growth 88, 148-158.

Dubors, M.. Daviaud, P., Rosin, O. \& Bergé, P. 1992 Travelling waves in pure fluids locally heated along wires. Physica D 61, 140-146.

FAROOQ, A. \& HomsY, G. M. 1994 Streaming flows due to g-jitter-induced natural convection. $J$. Fluid Mech. 271, 351-378.

Fowlis, W. W. \& RoberTs, G. O. 1986 Confinement of thermocapillary floating zone by uniform rotation. J. Cryst. Growth 74, 301-320.

HARLow. F. M. \& WELCH. J. E. 1965 Numerical calculation of time-dependent incompressible flow of fluid with a free surface. Phys. Fluids 8, 2182-2189.

Higuera. M., Nicolás. J. A. \& Vega. J. M. 1994 Linear oscillations of weakly dissipative axisymmetric liquid bridges. Phys. Fluids A 6, 438-450.

JURISH, M. \& LösER, W. 1990 Analysis of periodic non-rotational W striations in $M_{0}$ single crystals due to nonsteady thermocapillary convection. J. Cryst. Growth 102, 214-222.

KNobloch, E. \& PifRCE, R. D. 1997 On mean flows associated with travelling water waves. Preprint

LAN, C. W. \& KoU, S. 1991 Floating-zone crystal growth with a heated and immersed shaperexperiments. J. Cryst. Growth 108, 541-548.

Lee, C. P., Anilkumar, A. V. \& Wang, T. G. 1996 Streaming generated in a liquid bridge due to nonlinear oscillations driven by the vibration of an end-wall. Phvs. Fluids $8,3234-3246$.

Longuet-Higgins, M. S. 1953 Mass transport in water waves. Phil. Trans. R. Soc. Lond. A 245, $535-581$

Martel, C. \& Vega. J. M. 1996 Finite size effects near the onset of the oscillatory instability. Nonlinearity 9, 1129-1171.

Minkowycz, W. J.. Sparrow, E. M., Schneider, G. E. \& Pletcher, R. H. 1988 Handbook of Nimerical Heat Transfer. John Wiley \& Sons.

Mollot, D. J., Tsamopoulos, J., Chen, T. Y. \& Ashgriz, A. 1993 Nonlinear dynamics of capillary bridges: experiments. J. Fluid Mech. 255, 411-435. 
Nicolás, J. A., Rivas. D. \& VEGA, J. M. 1997 The interaction of thermocapillary convection and low-frequency vibration in nearly-inviscid liquid bridges. Z. Angew. Math. Phws. 48, 389-423.

NICOLÁs, J. A. \& VEGA, J. M. 1996 Weakly nonlinear oscillations of axisymmetric liquid bridges. $J$. Fluid Mech. 328, 95-128.

PaTankar, S. V. 1980 Numerical Heat Transfer and Fluid Flow. Hemisphere.

Phillips. O. M. 1977 Dynamics of the Upper Ocean, 2nd edn. Cambridge University Press.

Pierce. R. D. \& KNobloch, E. 1994 On the modulational stability of traveling and standing water waves. Phys. Fluids 6, 1177-1190.

RiLfy, N. 1992 Acoustic streaming about a cylinder in orthogonal beams. J. Fluid Mech. 242, 387-394.

Robertson JR., G. D. \& O'ConNor, D. 1988 Magnetic field effects on float-zone Si crystal growtl. III. Strong axial fields. J. Crvst. Growth $\mathbf{8 8}, 148-158$.

SANZ, A. 1985 The influence of the outer bath in the dynamics of axisymmetric liquid bridges. $J$. Fluid Mech. 156, 101-140.

Schlichting. H. 1932 Berechnung ebener periodischer Grenzschichtströmungen Phvs. Z. 33, 327335.

VAN Doormal. J. P. \& RaIThey, G. D. 1984 Enhancements of the SIMPLE method for predicting incompressible fluid flows. Numer. Heat Transfer 7, 147-163.

VINCE, J. M. \& DuboIs, M. 1992 Hot wire below the free surface of a liquid: Structural and dynamical properties of a secondary instability. Europhys. Lett. 20, 505-510.

Weidman, P., Afenchenko, V. O.. Ezersky, A. B.. Kiyashko. S. V. \& Rabinovich, M. I. 1997 The generation of two-dimensional vortices by transverse oscillation of a soap film. Gallery of Fluid Motion. Phys. Fluids 9, S2.

Whitham, G. B. 1974 Linear and Nonlinear Waves. Wiley.

YAN. B., Ingham, D. B. \& Morton, B. R. 1993 Streaming flow induced by an oscillating cascade of circular cylinders. J. Fluid Mech. 252, 147-171.

ZaPYANOV, Z., KOZHOUKHARONA, ZH. \& IORDANOVA, A. 1988 On the hydrodynamic interaction of two circular cylinders oscillating in a viscous fluid. Z. Angew. Math. Phys. 39, 204-220. 